\title{
A Triplectic Bi-Darboux Theorem and Para-Hypercomplex Geometry
}

\author{
IgOR A. BAtalin ${ }^{a}$ and Klaus Bering ${ }^{b}$ \\ ${ }^{a}$ I.E. Tamm Theory Division \\ P.N. Lebedev Physics Institute \\ Russian Academy of Sciences \\ 53 Leninsky Prospect \\ Moscow 119991 \\ Russia \\ ${ }^{b}$ Institute for Theoretical Physics \& Astrophysics \\ Masaryk University \\ Kotlářská 2 \\ CZ-611 37 Brno \\ Czech Republic
}

July 26, 2018

\begin{abstract}
We provide necessary and sufficient conditions for a bi-Darboux Theorem on triplectic manifolds. Here triplectic manifolds are manifolds equipped with two compatible, jointly non-degenerate Poisson brackets with mutually involutive Casimirs, and with ranks equal to $2 / 3$ of the manifold dimension. By definition bi-Darboux coordinates are common Darboux coordinates for two Poisson brackets. We discuss both the Grassmann-even and the Grassmann-odd Poisson bracket case. Odd triplectic manifolds are, e.g., relevant for $S p(2)$-symmetric field-antifield formulation. We demonstrate a one-to-one correspondence between triplectic manifolds and para-hypercomplex manifolds. Existence of bi-Darboux coordinates on the triplectic side of the correspondence translates into a flat Obata connection on the para-hypercomplex side.
\end{abstract}

MSC number(s): 37J99; 53D99; 55R10; 57R30; 58C50; 70S05.

Keywords: Poisson Bracket; Anti-bracket; Sp(2)-Symmetric Quantization; Darboux Theorem; Poincaré Lemma.

\footnotetext{
${ }^{a} \mathrm{E}-$ mail: batalin@lpi.ru $\quad{ }^{b} \mathrm{E}-$ mail: bering@physics .muni.cz
} 


\section{Contents}

1 Introduction $\quad 3$

1.1 General Remarks About Notation . . . . . . . . . . . . . . . . . . . . . 4

2 Bi-Poisson Structure $\quad 4$

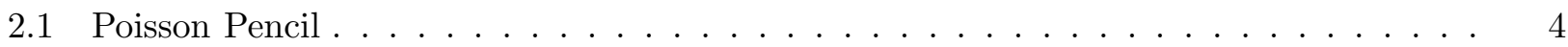

2.2 Global $G L(2)$ Covariance $\ldots \ldots \ldots \ldots \ldots \ldots \ldots \ldots$

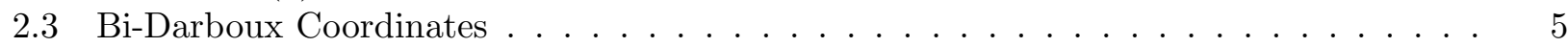

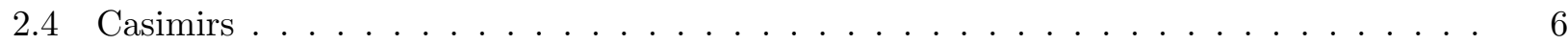

2.5 Fiber Bundle $\mathcal{M} \rightarrow \mathcal{N} \ldots \ldots \ldots \ldots \ldots \ldots$

2.6 Local Product Manifold $\mathcal{N} \ldots \ldots \ldots \ldots \ldots \ldots \ldots$

$2.7 E_{b j}^{a i}$ and $F^{a i j}$ Matrices $\ldots \ldots \ldots \ldots \ldots \ldots \ldots \ldots$

3 Bi-Darboux Theorem $\quad \mathbf{8}$

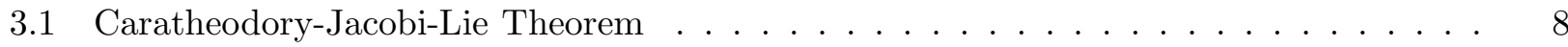

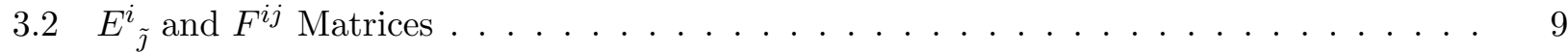

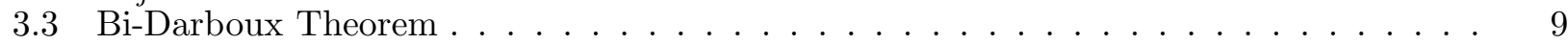

4 Closedness Conditions and Poincaré Lemma $\quad 10$

4.1 Closedness Condition for $E_{j}^{i} \ldots \ldots \ldots \ldots \ldots \ldots \ldots$

4.2 Closedness Condition for $\widetilde{E}^{\tilde{j}}{ }_{i} \ldots \ldots \ldots \ldots \ldots \ldots \ldots \ldots$

5 Canonical Transformations and Bundle Structure 12

5.1 Groupoids $\mathcal{G}, \mathcal{G}_{1}$ and $\mathcal{G}_{2} \ldots \ldots \ldots \ldots \ldots \ldots \ldots$

5.2 Canonical Transformations . . . . . . . . . . . . . . . . . . . 12

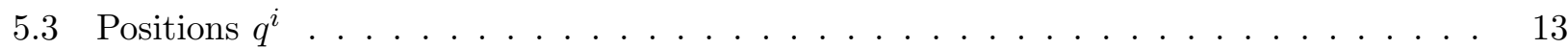

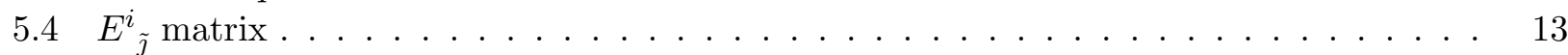

5.5 Para-Dolbeault Differentials . . . . . . . . . . . . . . . . . . 14

5.6 Presymplectic Potential $\vartheta \ldots \ldots \ldots \ldots \ldots \ldots \ldots \ldots$

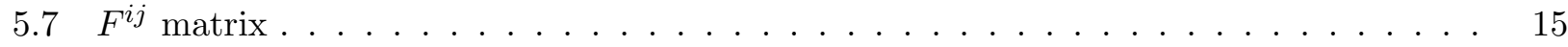

5.8 Gauge Bundle . . . . . . . . . . . . . . . . . . . . . 16

6 Proof of Bi-Darboux Theorem $r$

6.1 Factorization Condition . . . . . . . . . . . . . . . . 17

6.2 Bi-Poincaré Lemma . . . . . . . . . . . . . . . . . . . . . 17

$\begin{array}{llr}7 & \text { Bi-Canonical Transformations } & 18\end{array}$

8 Para-Hypercomplex Structure $r$

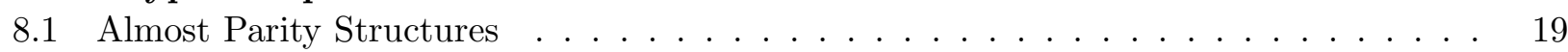

8.2 Parity Structures . . . . . . . . . . . . . . . . . . . . . . 19

8.2 .1 Parity Structure $\Sigma \ldots \ldots \ldots \ldots \ldots \ldots$

8.2 .2 Parity Structure $P \ldots \ldots \ldots \ldots \ldots \ldots \ldots \ldots$

8.3 Para-Hypercomplex Structure . . . . . . . . . . . . . . . . . . . 21

8.4 The Obata Connection $\nabla \ldots \ldots \ldots \ldots \ldots \ldots \ldots \ldots$

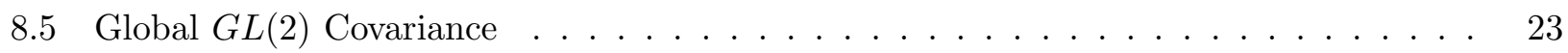

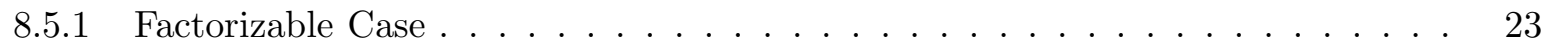


A Bi-Poincaré Lemma $\quad 24$

A.1 Algebra $\mathcal{A} \ldots \ldots \ldots \ldots \ldots \ldots \ldots \ldots$

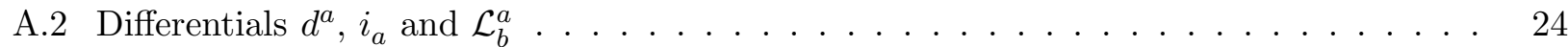

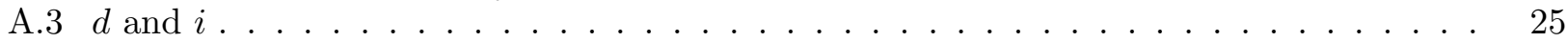

A.4 $\operatorname{sl}(2, \mathbb{C})$ Lie Algebra $\ldots \ldots \ldots \ldots \ldots \ldots \ldots \ldots$

A.5 Bi-Poincaré Lemma . . . . . . . . . . . . . . . . . . 25

A.6 $L$ and $\Lambda \ldots \ldots \ldots \ldots \ldots \ldots \ldots \ldots \ldots \ldots \ldots$

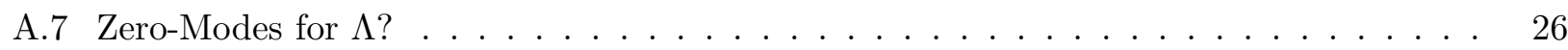

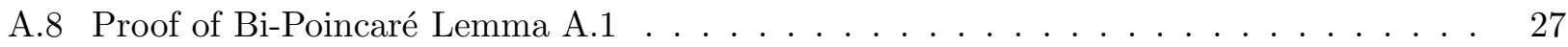

B Real Lie Groups

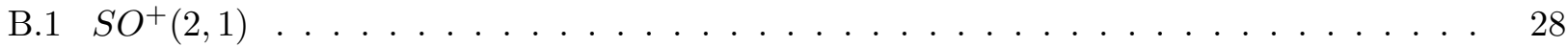

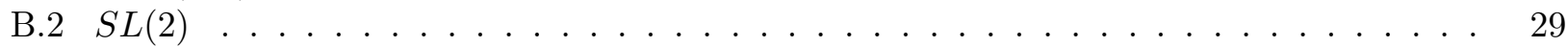

B.3 $G L(2) \ldots \ldots \ldots \ldots \ldots \ldots \ldots \ldots \ldots \ldots \ldots$

B.4 $S O^{+}(2,1) \cong \operatorname{Ad}(S L(2)) \ldots \ldots \ldots \ldots \ldots \ldots \ldots \ldots$

\section{Introduction}

A bi-Poisson supermanifold is a supermanifold equipped with two Poisson brackets. We shall here discuss both the case of two Grassmann-even Poisson brackets and the case of two Grassmann-odd Poisson brackets (also known as antibrackets).

Compatible Grassmann-even bi-Poisson structures have been studied extensively for more than thirty years in integrable systems $[1,2]$, usually with the extra assumption that at least one of the two Poisson structures are non-degenerate(=symplectic).

Compatible Grassmann-odd Poisson structures appear in the $S p(2)$-symmetric version $[3,4,5,6,7]$ of the field-antifield formulation $[8,9,10]$. This quantization scheme naturally live on a $3 n$-dimensional odd triplectic manifold $\mathcal{M}$. In particular, the total dimension of the underlying manifold $\mathcal{M}$ is a multiplum of 3. (In order to be as general as possible, we will here only be interested in the two antibrackets, and ignore the fact that the $S p(2)$-symmetric field-antifield formulation also contains two Grassmann-odd vector fields $V^{a}, a \in\{1,2\}$, which in turn would force the dimension of $\mathcal{M}$ to be a multiplum of 6 rather than 3.) Triplectic structures will in this paper refer to bi-Poisson structures that are jointly non-degenerate, with mutually involutive Casimirs, and with $2 / 3$ ranks, cf. Definition 2.3.

The main purpose of our paper is to investigate the possible existence of bi-Darboux coordinates for triplectic structures, i.e., if it is possible to locally bundle the coordinates of a triplectic manifold $\mathcal{M}$ into triplets $\left(q^{i}, p_{1 i}, p_{2 i}\right)$ of one position variable $q^{i}$ and two momentum variables $p_{1 i}, p_{2 i}$ each. The papers $[12,13]$ by Grigoriev and Semikhatov state the necessary and sufficient factorization condition (3.5) for the corresponding version of bi-Darboux Theorem, cf. Theorem 3.2, although without a complete* proof. We will here give a proof of the bi-Darboux Theorem 3.2 with the help of a new bi-Poincaré Lemma A.1. It turns out that the usual super-proof technique [11] for the standard Poincaré Lemma (which at its core is based on defining a suitable pairing between variables of opposite Grassmann-parity) is not applicable to the triplectic setting. Instead we give a proof of the bi-Poincaré Lemma A.1 with the help of $s l(2, \mathbb{C})$ representation theory.

${ }^{*}$ In detail, the existence of a function $H$ in eq. (3.17) of Ref. [12] relies implicitly on an un-proven version of the bi-Poincaré Lemma, which is covered in the case $E_{\tilde{\jmath}}^{i}=\delta_{\tilde{\jmath}}^{i}$ by our new bi-Poincaré Lemma A.1. 
The paper is organized as follows. Section 2 basic definitions and establishes notation. The main bi-Darboux Theorem 3.2 is stated in Subsection 3.3, and proved in Section 6. Sections 4-5 develop material and formalism needed in the proof. Section 7 contains a discussion of bi-canonical transformations, and Section 8 discusses a one-to-one correspondence between triplectic manifolds and para-hypercomplex manifolds. Para-hypercomplex geometry is a rapidly developing topic in differential geometry $[15,16,17,18]$ and in twisted supersymmetric $\mathcal{N}=(4,4)$ non-linear sigma-models [19]. Subsection 8.4 shows how para-hypercomplex supermanifolds are endowed with a unique Obata connection [14]. It turns out that the necessary and sufficient factorization condition (3.5) from the main bi-Darboux Theorem 3.2 is equivalent to that the Obata connection is flat. Finally, Appendix A contains a proof of bi-Poincaré Lemma A.1, while Appendix B lists some Lie group facts used in Section 8.

\subsection{General Remarks About Notation}

Adjectives from supermathematics such as "graded", "super", etc., are implicitly implied. The sign conventions are such that two exterior forms $\xi$ and $\eta$, of Grassmann-parity $\varepsilon_{\xi}, \varepsilon_{\eta}$ and of form-degree $p_{\xi}, p_{\eta}$, commute in the following graded sense

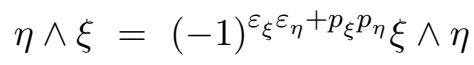

inside the exterior algebra. The exterior wedge symbol " $\wedge$ " is often not written explicitly, as it is redundant information that can be deduced from the Grassmann- and form-parity. The commutator $[F, G]$ and anticommutator $\{F, G\}_{+}$of two operators $F$ and $G$ are

$$
\begin{aligned}
& {[F, G]:=F G-(-1)^{\varepsilon_{F} \varepsilon_{G}+p_{F} p_{G} G F,}}
\end{aligned}
$$

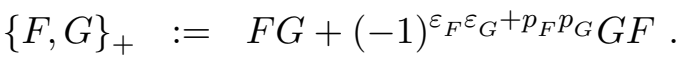

Note that in Section 4, Subsection 6.2, and Appendix A, there appear some objects $\eta_{i}$, $x_{3}^{i}$, etc., which are semantically referred to as "forms", although we will actually not assign any non-zero form-degree $p$ to them that affects their commutation properties (1.1).

\section{Bi-Poisson Structure}

\subsection{Poisson Pencil}

Let there be given a manifold $\mathcal{M}$ of dimension $3 n$ with two compatible Poisson brackets $\{\cdot, \cdot\}^{a}$, $a \in\{1,2\}$, of rank $2 n$, with common intrinsic Grassmann parity $\varepsilon$,

$$
\varepsilon\left(\{f, g\}^{a}\right)=\varepsilon_{f}+\varepsilon+\varepsilon_{g}, \quad f, g \in C^{\infty}(\mathcal{M}), \quad a \in\{1,2\},
$$

and with symmetry

$$
\{f, g\}^{a}=-(-1)^{\left(\varepsilon_{f}+\varepsilon\right)\left(\varepsilon_{g}+\varepsilon\right)}\{g, f\}^{a}, \quad f, g \in C^{\infty}(\mathcal{M}), \quad a \in\{1,2\} .
$$

In other words, the case $\varepsilon=0(\varepsilon=1)$ corresponds to a pair of even (odd) Poisson brackets, respectively. The word compatible means that any $\mathbb{R}$-linear combination of the two Poisson brackets $\{\cdot, \cdot\}^{a}, a \in$ $\{1,2\}$, is again a Poisson bracket, cf. Subsection 2.2. Alternatively, one says that the two Poisson

structures form a Poisson pencil. In particular, the two Poisson brackets satisfy a symmetrized Jacobi identity

$$
\sum_{\text {cycl. } f, g, h}(-1)^{\left(\varepsilon_{f}+\varepsilon\right)\left(\varepsilon_{h}+\varepsilon\right)}\left\{\{f, g\}^{\{a}, h\right\}^{b\}}=0, \quad f, g, h \in C^{\infty}(\mathcal{M}), \quad a, b \in\{1,2\},
$$


which contains the Jacobi identity for each Poisson brackets, and a six-term mixed Jacobi identity.

The symmetrized Jacobi identity (2.3) is a very important geometrical input. A good part of the following Sections 2-4 will deal with extracting exhaustively the huge amount of geometric information that it contains.

\subsection{Global $G L(2)$ Covariance}

The construction must behave covariantly under the group ${ }^{\dagger} G L(2)=S L(2) \times \mathbb{R}^{\times}$of global rotations of the two Poisson brackets,

$$
\{\cdot,\}^{a} \rightarrow\{\cdot, \cdot\}^{\prime b}=\{\cdot, \cdot\}^{a}\left(g^{-1}\right)_{a}^{b}, \quad g \in G L(2) .
$$

where the group $G L(2)$ by definition acts from left. It turns out that the overall scaling group ${ }^{\ddagger}$ $\mathbb{R}^{\times} \equiv \mathbb{R} \backslash\{0\}$ acts trivially (basically because it belongs to the center of $G L(2)$ ), so that only the $S L(2)=S p(2)$ part is interesting. We should stress that we here do not a priori assume the existence of an "intrinsic" group action "." $: S L(2) \times \mathcal{M} \rightarrow \mathcal{M}$ on the manifold $\mathcal{M}$, and hence a group action "." : $S L(2) \times C^{\infty}(\mathcal{M}) \rightarrow C^{\infty}(\mathcal{M})$ of functions defined as

$$
(g . f)(z):=f\left(g^{-1} . z\right), \quad f \in C^{\infty}(\mathcal{M}), \quad g \in S L(2), \quad z \in \mathcal{M}
$$

that is compatible

$$
g \cdot\{f, h\}^{b}=\{g \cdot f, g \cdot h\}^{a}\left(g^{-1}\right)_{a}^{b}, \quad f, h \in C^{\infty}(\mathcal{M}), \quad g \in S L(2),
$$

with the rotations (2.4) of the two Poisson brackets. See also Subsection 8.5.

\subsection{Bi-Darboux Coordinates}

General local coordinates are called $z^{A}, A \in\{1, \ldots, 3 n\}$, and they are assumed to have definite Grassmann parity $\varepsilon_{A} \equiv \varepsilon\left(z^{A}\right)$. (More precisely, the local coordinates $z^{A}$ are functions on an open neighborhood $\mathcal{U} \subseteq \mathcal{M}$, and usually not globally defined. Nevertheless, we will often, with a slight misuse of notation, not explicitly mention the neighborhood $\mathcal{U}$, and write $z^{A} \in C^{\infty}(\mathcal{M}), d z^{A} \in$ $\Gamma\left(T^{*} \mathcal{M}\right)$, etc., rather than $z^{A} \in C^{\infty}(\mathcal{U}), d z^{A} \in \Gamma\left(\left.T^{*} \mathcal{M}\right|_{\mathcal{U}}\right)$, respectively.)

Definition 2.1 Bi-Darboux coordinates (or bi-canonical coordinates) for the two Poisson brackets $\{\cdot, \cdot\}^{a}, a \in\{1,2\}$, are a common set of local Darboux coordinates $\left\{z^{A}\right\}=\left\{q^{i} ; p_{a j}\right\}, i, j \in$ $\{1, \ldots, n\}, a \in\{1,2\}$, with Grassmann parities $\varepsilon_{i} \equiv \varepsilon\left(q^{i}\right)$ and $\varepsilon\left(p_{a j}\right)=\varepsilon_{j}+\varepsilon$, such that

$$
\{f, g\}^{a}=f\left(\frac{\overleftarrow{\partial^{r}}}{\partial q^{i}} \frac{\overrightarrow{\partial^{\ell}}}{\partial p_{a i}}-(-1)^{\varepsilon_{i}(1-\varepsilon)} \frac{\overleftarrow{\partial^{r}}}{\partial p_{a i}} \frac{\overrightarrow{\partial^{\ell}}}{\partial q^{i}}\right) g, \quad f, g \in C^{\infty}(\mathcal{M}), \quad a \in\{1,2\}
$$

\footnotetext{
${ }^{\dagger}$ The matrix $g_{a}{ }^{b}$ for the group element $g \in G L(2)$ is unconventionally written with its indices upside-down. For instance, the transposed matrix is written as $\left(g^{T}\right)^{a}{ }_{b}:=g_{b}{ }^{a}$.

$\ddagger$ The scaling group $\mathbb{R}^{\times}$is absent in the $S p(2)$-symmetric field-antifield formulation $[3,4,5,6,7]$ because of explicit appearances of the Levi-Civita $\epsilon^{a b}$ tensor. See also Appendix B.
} 


\subsection{Casimirs}

Definition 2.2 A local function $f \in C^{\infty}(\mathcal{U}), \mathcal{U} \subseteq \mathcal{M}$, is by definition a Casimir $^{\S}$ for the a'th Poisson bracket $\{\cdot, \cdot\}^{a}$ if the corresponding local Hamiltonian vector field $X_{f}^{a}:=\{f, \cdot\}^{a}=0$ vanishes identically.

The subalgebra (more correctly, subsheaf) of Casimirs for the first (second) Poisson bracket is denoted $\mathcal{C}_{2}\left(\mathcal{C}_{1}\right)$, respectively. (Notice the reversed labeling convention!) The $2 n$ rank condition means that the subalgebra $\mathcal{C}_{a} \subseteq C^{\infty}(\mathcal{M}), a \in\{1,2\}$, is locally generated by $n$ independent Casimir coordinates $\xi_{a i}, i \in\{1, \ldots, n\}$. (The notation $\xi_{a i}$ is a bit misleading in the sense that $\xi_{a i}$ does not necessarily transform as an $S L(2)$ doublet under $S L(2)$ rotations of the " $a$ " index.) For fixed $a \in\{1,2\}$, the set of local Casimir coordinates $\xi_{a i}$ is unique up to reparametrizations $\xi_{a i} \rightarrow \xi_{a j}^{\prime}=\xi_{a j}^{\prime}\left(\xi_{a}\right)$.

The above reversed labeling convention implies that $\left\{\cdot, \xi_{a i}\right\}^{b}$ is diagonal in the ${ }_{a}{ }^{b}$ indices. (This choice of labeling convention is necessary, so that, e.g., the formula (2.7) for bi-Darboux coordinates becomes manifestly $G L(2)$ covariant under the identification $p_{a i}=\xi_{a i}$.)

The two Poisson brackets $\{\cdot, \cdot\}^{a}, a \in\{1,2\}$, are furthermore assumed to have the following properties.

1. They are jointly non-degenerate, which means that they have no common Casimirs $\mathcal{C}_{1} \cap \mathcal{C}_{2} \subseteq\{0\}$.

2. They have mutually involutive Casimirs, which means that the Casimirs with respect to one bracket are in involution with respect to the other bracket, and vice-versa. In other words,

$$
\{f, g\}^{a}=0, \quad f, g \in \mathcal{C}_{a}, \quad a \in\{1,2\} .
$$

This can be written compactly as $\left\{\mathcal{C}_{a}, \mathcal{C}_{a}\right\}^{a} \subseteq\{0\}$; or equivalently, in local Casimir coordinates,

$$
\left\{\xi_{a i}, \xi_{a j}\right\}^{a}=0, \quad i, j \in\{1, \ldots, n\}, \quad a \in\{1,2\} .
$$

In fact, it follows from eq. (2.9) and the Casimir property, that $\left\{\mathcal{C}_{a}, \mathcal{C}_{b}\right\}^{c} \subseteq\{0\}$, or equivalently,

$$
\left\{\xi_{a i}, \xi_{b j}\right\}^{c}=0, \quad i, j \in\{1, \ldots, n\}, \quad a, b, c \in\{1,2\}
$$

Definition 2.3 A triplectic manifold $\left(\mathcal{M} ;\{\cdot, \cdot\}^{a}\right)$ is a $3 n$-dimensional manifold $\mathcal{M}$ equipped with two Poisson brackets $\{\cdot, \cdot\}^{a}, a \in\{1,2\}$,

1. that both have rank $2 n$,

2. that have common intrinsic Grassmann parity $\varepsilon$,

3. that are compatible,

4. that are jointly non-degenerate,

5. and that have mutually involutive Casimirs.

\footnotetext{
${ }^{\S}$ Casimirs are called marked functions in Ref. [12], Ref. [13] and Ref. [20].

`Other names are mutually flat or mutually commutative, cf. Ref. [12], Ref. [13] and Ref. [20].
} 


\section{$2.5 \quad$ Fiber Bundle $\mathcal{M} \rightarrow \mathcal{N}$}

We assume from now on that $\left(\mathcal{M} ;\{\cdot, \cdot\}^{a}\right)$ is a $3 n$-dimensional triplectic manifold. For each Poisson bracket $\{\cdot, \cdot\}^{a}, a \in\{1,2\}$, there exists an integrable distribution $\Delta^{a}=T\left(\mathcal{M}_{a}\right) \subseteq T \mathcal{M}$, generated by the Hamiltonian vector fields $X_{f}^{a}:=\{f, \cdot\}^{a}, f \in C^{\infty}(\mathcal{M})$. The distribution $\Delta^{a}=T\left(\mathcal{M}_{a}\right)$ gives rise to a $2 n$-foliation of $\mathcal{M}$ called symplectic leaves. Locally, the $2 n$-dimensional symplectic leaves are labeled by $n$ constants $\xi_{a i}^{(0)}, i \in\{1, \ldots, n\}$,

$$
\begin{aligned}
& \left.\mathcal{M}_{1}\left(\xi_{2 i}^{(0)}\right)\right|_{\mathcal{U}}:=\left\{z \in \mathcal{U} \mid \forall i \in\{1, \ldots, n\}: \xi_{2 i}=\xi_{2 i}^{(0)}\right\} \\
& \left.\mathcal{M}_{2}\left(\xi_{1 i}^{(0)}\right)\right|_{\mathcal{U}}:=\left\{z \in \mathcal{U} \mid \forall i \in\{1, \ldots, n\}: \xi_{1 i}=\xi_{1 i}^{(0)}\right\}
\end{aligned}
$$

The $n$-dimensional submanifolds

$$
\mathcal{M}_{1}\left(\xi_{2 i}^{(0)}\right) \cap \mathcal{M}_{2}\left(\xi_{j 1}^{(0)}\right)
$$

of intersecting symplectic leaves, are again leaves that constitute an $n$-foliation of $\mathcal{M}$. (The $n$-leaves (2.12) are not necessarily Lagrangian/involutive, due to possible presence of $F^{a i j}$ matrices (2.15), cf. Section 2.7.)

Let us collectively call all the $2 n$ Casimir coordinates for $\xi^{I}=\xi_{a i}$, where $I \in\{1, \ldots, 2 n\}, i \in\{1, \ldots, n\}$, $a \in\{1,2\}$. Let the local leaf coordinates (i.e., the coordinates that parametrize a single $n$-leaf) be $q^{i}$, with Grassmann parity $\varepsilon_{i} \equiv \varepsilon\left(q^{i}\right), i \in\{1, \ldots, n\}$, in such a way that $\left\{z^{A}\right\}=\left\{q^{i} ; \xi^{I}\right\}$ constitutes a local coordinate system for the total space $\mathcal{M}$.

As we shall see in Section 5.3, there exists an atlas of distinguished ${ }^{\|}$coordinate systems $\left\{z^{A}\right\}=\left\{q^{i} ; \xi^{I}\right\}$ for $\mathcal{M}$, in-which the leaf coordinates $q^{i} \rightarrow q^{j}$ transform affinely under coordinate transformations $z^{A} \longrightarrow z^{B}=z^{B}(z)$. In other words, an $n$-leaf (2.12) is always (a subsets of) an $n$-dimensional affine space.

For this reason, we shall from now on assume the following model for the $3 n$-dimensional manifold $\mathcal{M}$ (which locally captures the general situation).

Assumption 2.4 (Fiber bundle) The triplectic manifold $\mathcal{M}$ is globally a (not necessarily affine) fiber bundle $\mathcal{M} \rightarrow \mathcal{N}$ over a $2 n$-dimensional base manifold $\mathcal{N}$ with local base coordinates $\xi^{I}, I \in$ $\{1, \ldots, 2 n\}$ consisting of Casimirs. (To be more precise, a local Casimir coordinate in $\mathcal{M}$ is a pullback $\pi^{*} \xi_{a i}:=\xi_{a i} \circ \pi$ of a local coordinate $\xi^{I}$ on $\mathcal{N}$ via the canonical projection map $\pi: \mathcal{M} \rightarrow \mathcal{N}$.)

The $n$-dimensional fibers have local fiber coordinates $q^{i}, i \in\{1, \ldots, n\}$.

\subsection{Local Product Manifold $\mathcal{N}$}

The $2 n$-dimensional base manifold $\mathcal{N}$ has two $n$-foliations with $n$-dimensional leaves

$$
\mathcal{N}_{1}\left(\xi_{2 i}^{(0)}\right):=\pi\left(\mathcal{M}_{1}\left(\xi_{2 i}^{(0)}\right)\right), \quad \mathcal{N}_{2}\left(\xi_{1 i}^{(0)}\right):=\pi\left(\mathcal{M}_{2}\left(\xi_{1 i}^{(0)}\right)\right)
$$

respectively. Here $\pi: \mathcal{M} \rightarrow \mathcal{N}$ is the canonical projection map, and here $\xi_{a i}^{(0)}$ are constants that label the leaves. The $n$-dimensional tangent space $T\left(\mathcal{N}_{a}\right)=\pi_{*}\left(\Delta^{a}\right)$ is an integrable distribution $\subseteq T \mathcal{N}$. All of this implies that $\mathcal{N}$ is a local product manifold, which means that there exists an atlas

\footnotetext{
" A distinguished element of a set means an element that has an extra property, which depends on context.
} 
of distinguished coordinate systems $\left\{\xi^{I}\right\}=\left\{\xi_{1 i} ; \xi_{2 i}\right\}$ such that a general coordinate transformation $\xi^{I} \longrightarrow \xi^{\prime J}=\xi^{J}(\xi)$ between two distinguished coordinate systems splits in two sectors,

$$
\xi_{1 i} \longrightarrow \xi_{j 1}^{\prime}=\xi_{j 1}^{\prime}\left(\xi_{1}\right), \quad \xi_{2 i} \longrightarrow \xi_{j 2}^{\prime}=\xi_{j 2}^{\prime}\left(\xi_{2}\right)
$$

\section{7 $\quad E^{a i}{ }_{b j}$ and $F^{a i j}$ Matrices}

Observation 2.5 In coordinates of the form $\left\{z^{A}\right\}=\left\{q^{i} ; \xi^{I}\right\}$, a fundamental Poisson bracket $\left\{z^{A}, z^{B}\right\}^{a}$ can only be non-zero if at least one of the entries $z^{A}$ or $z^{B}$ is a $q^{i}$ variable.

In other words, there are no traces of the bi-Poisson structure on the base manifold $\mathcal{N}$ itself, cf. eq. (2.10). The only remaining non-zero fundamental Poisson brackets $\left\{z^{A}, z^{B}\right\}^{a}$ are given by

$$
E_{b j}^{a i}:=\left\{q^{i}, \xi_{b j}\right\}^{a}, \quad F^{a i j}:=\left\{q^{i}, q^{j}\right\}^{a}, \quad i, j \in\{1, \ldots, n\}, \quad a, b \in\{1,2\} .
$$

In fact, one can say more. Note that the $2 n \times 2 n$ matrix $E^{a i}{ }_{b j}$ is diagonal in the ${ }_{b}{ }_{b}$ indices, due to the Casimir property, and therefore only consists of two $n \times n$ block matrices, apart from trivial zero entries. Thus the matrices (2.15) effectively only contain four quadratic $n \times n$ block matrices, where the third and fourth $n \times n$ block matrix come from the $2 \times n \times n$ matrix $F^{a i j}$. The $2 n$ rank condition for $\{\cdot, \cdot\}^{a}$ yields the following Observation 2.6.

Observation 2.6 The two $E^{a i}{ }_{a j}$ block matrices are invertible, $a \in\{1,2\}$.

Definition 2.7 The a'th Poisson bracket $\{\cdot, \cdot\}^{a}$ is said to be on Darboux form (or canonical form) if $E^{a i}{ }_{a j}=\delta_{j}^{i}$ and $F^{a i j}=0$.

\section{Bi-Darboux Theorem}

\subsection{Caratheodory-Jacobi-Lie Theorem}

We now continue dissecting the symmetrized Jacobi identity (2.3) in a triplectic context. To proceed, it is convenient to break the manifest $1 \leftrightarrow 2$ labeling symmetry between the two Poisson brackets $\{\cdot, \cdot\}^{a}, a \in\{1,2\}$. We will rename the Casimirs $\xi_{a i}$ as

$$
p_{i} \equiv \xi_{1 i}, \quad c_{\tilde{\jmath}} \equiv \xi_{2 \tilde{\jmath}}, \quad i, \tilde{\jmath} \in\{1, \ldots, n\}
$$

for reasons that will soon become clear.

According to (a superversion of) the Caratheodory-Jacobi-Lie Theorem [21] (with the Casimir $c$ variables as passive spectator parameters), it is possible to introduce position coordinates $q^{i}, i \in\{1, \ldots, n\}$, such that the first Poisson bracket $\{\cdot, \cdot\}^{1}$ is on Darboux form

$$
E^{1 i}{ }_{1 j}=\left\{q^{i}, p_{j}\right\}^{1}=\delta_{j}^{i}, \quad F^{1 i j}=\left\{q^{i}, q^{j}\right\}^{1}=0, \quad i, j \in\{1, \ldots, n\} .
$$

We emphasize that the Darboux form for the first Poisson bracket can be achieved without changing the momenta $p_{i}$ and the Casimirs $c_{\tilde{\jmath}}$. The Grassmann parity of the momentum variables $p_{i}$ must be $\varepsilon\left(p_{i}\right)=\varepsilon_{i}+\varepsilon$. 


\section{$3.2 E^{i}$ and $F^{i j}$ Matrices}

The only remaining non-zero fundamental brackets $\left\{z^{A}, z^{B}\right\}^{2}$ for the second Poisson bracket are given by two quadratic $n \times n$ matrices

$$
E^{i}{ }_{\tilde{\jmath}}:=E^{2 i}{ }_{2 \tilde{\jmath}}:=\left\{q^{i}, c_{\tilde{\jmath}}\right\}^{2}, \quad F^{i j}:=F^{2 i j}:=\left\{q^{i}, q^{j}\right\}^{2}, \quad i, j, \tilde{\jmath} \in\{1, \ldots, n\} .
$$

The Grassmann parities are $\varepsilon\left(E_{\tilde{\jmath}}^{i}\right)=\varepsilon\left(p_{i}\right)+\varepsilon\left(c_{\tilde{\jmath}}\right)$ and $\varepsilon\left(F^{i j}\right)=\varepsilon_{i}+\varepsilon+\varepsilon_{j}$, respectively.

The second Poisson bracket $\{\cdot, \cdot\}^{2}$ is on Darboux form if $E_{\tilde{\jmath}}^{i}=\delta_{\tilde{j}}^{i}$ and $F^{i j}=0$, and in that case we would have achieved a bi-Darboux form of the two Poisson brackets.

If one inspects the six-term mixed Jacobi identity (2.3) in the $q p c$ and $q q p$ sectors, it turns out that five of the six terms vanish because of eq. (3.2) or the Casimir property. Hence the remaining lone term must vanish as well,

$$
\left\{\left\{q^{i}, c_{\tilde{\jmath}}\right\}^{2}, p_{k}\right\}^{1}=0, \quad\left\{\left\{q^{i}, q^{j}\right\}^{2}, p_{k}\right\}^{1}=0,
$$

respectively. Equation (3.4) implies that the matrices $E_{\tilde{j}}^{i}=E_{\tilde{j}}^{i}(p, c)$ and $F^{i j}=F^{i j}(p, c)$ are independent of the $q$ variables. This yields the following Observation 3.1.

Observation 3.1 In coordinates $\left\{z^{A}\right\}=\left\{q^{i} ; \xi^{I}\right\}$, where the first Poisson bracket $\{\cdot, \cdot\}^{1}$ is on Darboux form (3.2), the fundamental Poisson brackets $\left\{z^{A}, z^{B}\right\}^{a}$ do not depend on the $q$ variables.

In other words, the fundamental Poisson brackets $\left\{z^{A}, z^{B}\right\}^{a}$ live down in the base manifold $\mathcal{N}$.

\subsection{Bi-Darboux Theorem}

We are now ready to state the bi-Darboux Theorem 3.2.

Theorem 3.2 (Bi-Darboux Theorem) A necessary and sufficient condition for a triplectic manifold $\left(\mathcal{M} ;\{\cdot, \cdot\}^{a}\right)$ to have bi-Darboux coordinates is a local factorization** (or separation of variables) condition for the $E^{i}{ }_{k}$ matrix (3.3), i.e., there should exist matrices $P_{j}^{i}=P_{j}^{i}(p)$ and $C^{j}{ }_{k}=$ $C^{j}{ }_{k}(c)$ such that

$$
E(p, c)=P(p) C(c) \quad \Leftrightarrow \quad E_{k}^{i}(p, c)=P_{j}^{i}(p) C_{k}^{j}(c)
$$

We will give a proof of the bi-Darboux Theorem 3.2 in Section 6. The factorization (3.5) is unique up to a constant invertible matrix $K_{j}^{i}$, i.e.,

$$
P \longrightarrow P K, \quad C \longrightarrow K^{-1} C
$$

because of separation of the $p$ and $c$ variables. The corresponding differential factorization condition reads

$$
\frac{\overrightarrow{\partial^{\ell}}}{\partial p_{i}}\left[\left(\frac{\overrightarrow{\partial^{\ell}}}{\partial c_{\tilde{\jmath}}} \widetilde{E}\right) E\right]=0 \quad \Leftrightarrow \quad \frac{\overrightarrow{\partial^{\ell}}}{\partial c_{\tilde{\jmath}}}\left[\left(\frac{\overrightarrow{\partial^{\ell}}}{\partial p_{i}} E\right) \widetilde{E}\right]=0
$$

\footnotetext{
${ }^{* *}$ Theorem 3.2 is essentially stated as Theorem 4.3 in Ref. [13]. A factorizable $E^{i}{ }_{k}$ matrix (3.5) is referred to as a reducible matrix in Ref. [12] and Ref. [13]. Those papers rely on additional structures (the odd vector fields $V^{a}$ ), which is not used here in order to be as general as possible.
} 
where $\widetilde{E}:=E^{-1}$ denotes the inverse matrix; see also eq. (4.16). The differential factorization condition (3.7) is equivalent to that the Obata connection $\nabla$ should be flat, see Theorem 8.4.

A 3-dimensional example. Let the triplectic manifold be $\mathcal{M}=\left\{\left(q^{1}, p_{1}, c_{1}\right) \in \mathbb{R}^{3} \mid p_{1}+c_{1} \neq 0\right\}$ with global coordinates $\left\{q^{1} ; p_{1} ; c_{1}\right\}$. Let the first Poisson bracket $\{\cdot, \cdot\}^{1}$ be on Darboux form, and let the second Poisson bracket $\{\cdot, \cdot\}^{2}$ have $E_{k}^{i}$ matrix (3.3) equal to $E^{1}{ }_{1}=\left\{q^{1}, c_{1}\right\}^{2}=p_{1}+c_{1}$. This is a Poisson pencil (2.3) that does not satisfy the factorization condition (3.5), and hence no bi-Darboux coordinates exist.

\section{Closedness Conditions and Poincaré Lemma}

In this Section 4, we in particular derive the eqs. (4.10), (4.13) and (4.17), which will be needed in Sections 5-6.

Let $\eta_{i}, i \in\{1, \ldots, n\}$, be new auxiliary local ${ }^{\dagger \dagger}$ "one-form" variables of Grassmann parity $\varepsilon\left(\eta_{i}\right)=$ $\varepsilon_{i}+1-\varepsilon=\varepsilon\left(p_{i}\right)+1$. The Poisson brackets $\{\cdot, \cdot\}^{a}, a \in\{1,2\}$, are now trivially extended such that the $\eta$ variables are new Casimirs for both Poisson brackets. Define Grassmann-odd differentials as

$$
\begin{gathered}
d^{a}:=\eta_{i}\left\{q^{i},\right\}^{a}=\left\{\eta_{i} q^{i}, \cdot\right\}^{a}, \quad \varepsilon\left(d^{a}\right)=1, \quad a \in\{1,2\}, \\
d^{1}=\eta_{i} \frac{\overrightarrow{\partial^{\ell}}}{\partial p_{i}}, \quad d^{2}=\eta_{i} E^{i}{ }_{\tilde{\jmath}} \frac{\overrightarrow{\partial^{\ell}}}{\partial c_{\tilde{\jmath}}}+\eta_{i} F^{i j} \frac{\overrightarrow{\partial^{\ell}}}{\partial q^{j}} .
\end{gathered}
$$

The super-commutator reads

$$
\left[d^{a}, d^{b}\right]=d^{\{a} d^{b\}}=\frac{1}{2}(-1)^{\varepsilon\left(\eta_{j}\right)} \eta_{j} \eta_{i}\left\{\left\{q^{i}, q^{j}\right\}^{\{a}, \cdot\right\}^{b\}}=\left\{\beta^{\{a}, \cdot\right\}^{b\}}, \quad a, b \in\{1,2\} .
$$

Here

$$
\beta^{a}:=\frac{1}{2}(-1)^{\varepsilon\left(\eta_{j}\right)} \eta_{j} \eta_{i}\left\{q^{i}, q^{j}\right\}^{a}=\frac{1}{2} \eta_{i}\left\{q^{i}, q^{j}\right\}^{a} \eta_{j}(-1)^{\varepsilon\left(\eta_{j}\right) \varepsilon}, \quad a \in\{1,2\},
$$

are two-forms. The super-commutator (4.3) vanishes if we restrict the differentials $d^{a}$ to act on an algebra $\mathcal{F}$ of functions $f=f(p, c, \eta)$ that do not depend on the $q$ variables. This is basically because $\left\{q^{i}, q^{j}\right\}^{a} \in \mathcal{F}$ does not depend on the $q$ 's, cf. Observation 3.1. Concretely, the $q^{j}$ differentiation in eq. (4.2) becomes irrelevant. The two-forms $\beta^{a} \in \mathcal{F}$ and the one-forms

$$
\alpha_{\tilde{\jmath}}^{a}:=d^{a} c_{\tilde{\jmath}} \in \mathcal{F}, \quad \tilde{\jmath} \in\{1, \ldots, n\}, \quad a \in\{1,2\},
$$

both belong to $\mathcal{F}$. It follows from the symmetrized Jacobi identity (2.3) in the $q q q$ and $q q c$ sectors that

$$
d^{\{a} \beta^{b\}}=0, \quad a, b \in\{1,2\}
$$

and

$$
d^{\{a} \alpha_{\tilde{\jmath}}^{b\}}=d^{\{a} d^{b\}} c_{\tilde{\jmath}}=0, \quad a, b \in\{1,2\},
$$

respectively. Now, we already know from Section 3.1 that the first structures

$$
\beta^{1}=0 \quad \text { and } \quad \alpha_{\tilde{\jmath}}^{1}=0
$$

\footnotetext{
${ }^{\dagger}$ Since we are only interested in a bi-Darboux Theorem, we may work locally in coordinates. The word local refers to a sufficiently small open neighborhood $\mathcal{U}$. We will not repeat this point further in the text. Concretely, we will ignore extending some local constructions to a global setting.
} 
are zero, so we are really only interested in the second structures

$$
\beta^{2}=\frac{1}{2} \eta_{i} F^{i j} \eta_{j}(-1)^{\varepsilon\left(\eta_{j}\right) \varepsilon} \quad \text { and } \quad \alpha_{\tilde{\jmath}}^{2}=\eta_{i} E_{\tilde{\jmath}}^{i}
$$

It follows that $\beta^{2}$ and $\alpha_{\tilde{\jmath}}^{2}$ are $d^{a}$-closed,

$$
d^{a} \beta^{2}=0, \quad d^{a} \alpha_{\tilde{\jmath}}^{2}=0, \quad a \in\{1,2\}
$$

\subsection{Closedness Condition for $E^{i}$}

The mixed closedness condition $d^{1} \alpha_{\tilde{\jmath}}^{2}=0$ reads explicitly,

$$
\left(\frac{\overrightarrow{\partial^{\ell}}}{\partial p_{i}} E^{k}\right)=(-1)^{\varepsilon\left(p_{i}\right) \varepsilon\left(p_{k}\right)}(i \leftrightarrow k) .
$$

By the standard Poincaré Lemma for $d^{1}$, there exist zero-forms $A_{\tilde{\jmath}}=A_{\tilde{\jmath}}(p, c) \in \mathcal{F}$ such that

$$
\alpha_{\tilde{\jmath}}^{2}=d^{1} A_{\tilde{\jmath}}
$$

or explicitly,

$$
E_{\tilde{\jmath}}^{i}=\left(\frac{\overrightarrow{\partial^{\ell}}}{\partial p_{i}} A_{\tilde{\jmath}}\right)
$$

\subsection{Closedness Condition for $\widetilde{E}^{\tilde{j}}{ }_{i}$}

2) The closedness condition $d^{2} \alpha_{\tilde{\jmath}}^{2}=\left(d^{2}\right)^{2} c_{\tilde{\jmath}}=0$ reads explicitly,

$$
E_{\tilde{m}}^{i}\left(\frac{\overrightarrow{\partial^{\ell}}}{\partial c_{\tilde{m}}} E_{\tilde{k}}^{j}\right)=(-1)^{\varepsilon\left(p_{i}\right) \varepsilon\left(p_{j}\right)}(i \leftrightarrow j),
$$

or equivalently,

$$
\frac{\partial^{\ell}}{\partial c_{\tilde{\imath}}} \widetilde{E}_{k}^{\tilde{\jmath}}=(-1)^{\varepsilon\left(c_{\tilde{\imath}}\right) \varepsilon\left(c_{\tilde{\jmath}}\right)}(\tilde{\imath} \leftrightarrow \tilde{\jmath}),
$$

where we have defined the inverse matrix

$$
\widetilde{E}_{i}^{\tilde{\jmath}}:=\left(E^{-1}\right)^{\tilde{\jmath}}{ }_{i},
$$

cf. Observation 2.6. By the standard Poincaré Lemma, there exist functions $\widetilde{A}_{i}=\widetilde{A}_{i}(p, c) \in \mathcal{F}$, so that

$$
\widetilde{E}_{i}^{\tilde{\jmath}}=\left(\frac{\overrightarrow{\partial^{\ell}}}{\partial c_{\tilde{\jmath}}} \widetilde{A}_{i}\right)
$$




\section{Canonical Transformations and Bundle Structure}

\subsection{Groupoids $\mathcal{G}, \mathcal{G}_{1}$ and $\mathcal{G}_{2}$}

It is very restricted what local coordinate transformations $z^{A} \rightarrow z^{\prime B}=z^{\prime B}(z)$ can still be performed without spoiling the progress so far in the attempt to achieve bi-Darboux coordinates. They are given by the following groupoid $(\mathcal{G} ; \circ)$.

Definition 5.1 Let $(\mathcal{G} ; \circ)$ be the groupoid of local coordinate transformations $z^{A} \rightarrow z^{\prime B}=z^{B}(z)$ that satisfy the following conditions.

- They preserve the Darboux form (3.2) of the first Poisson bracket $\{\cdot, \cdot\}^{1}$.

- They at most reparametrize the Casimirs $p_{i} \rightarrow p_{j}^{\prime}=p_{j}^{\prime}(p)$ and $c_{\tilde{\imath}} \rightarrow c_{\tilde{\jmath}}^{\prime}=c_{\tilde{\jmath}}^{\prime}(c)$.

Definition 5.2 Let $\mathcal{G}_{1} \subseteq \mathcal{G}$ be the subgroupoid of local coordinate transformations $z^{A} \rightarrow z^{\prime B}=z^{\prime B}(z)$ that do not transform the second set of Casimirs $c_{\tilde{\imath}}^{\prime}=c_{\tilde{i}}$.

Definition 5.3 Let $\mathcal{G}_{2} \subseteq \mathcal{G}$ be the subgroupoid of local coordinate transformations $z^{A} \rightarrow z^{\prime B}=z^{\prime B}(z)$ that do not transform $q^{\prime i}=q^{i}$ nor $p_{j}^{\prime}=p_{j}$ but do only reparametrize the second Casimirs $c_{\tilde{\imath}} \rightarrow c_{\tilde{\jmath}}^{\prime}=$ $c_{\tilde{\jmath}}^{\prime}(c)$.

The two subgroupoids $\mathcal{G}_{1}$ and $\mathcal{G}_{2}$ commute, and each coordinate transformations $z^{A} \rightarrow z^{B}=z^{B}(z)$ in $\mathcal{G}$ may be uniquely factorized in two coordinate transformations from $\mathcal{G}_{1}$ and $\mathcal{G}_{2}$, respectively.

\subsection{Canonical Transformations}

Let us first consider a coordinate transformation $z^{A} \rightarrow z^{\prime B}=z^{\prime B}(z)$ in just $\mathcal{G}_{1}$, which preserves the first Poisson bracket on Darboux form and does not transform the Casimir $c$ variables. In other words, it is a canonical transformation (with respect to the first Poisson bracket and with the Casimir $c$ variables as passive spectator parameters). As mentioned in Ref. [22], if the canonical transformation is sufficiently close to the identity, there exists a corresponding generator $F_{3}=F_{3}\left(q^{\prime}, p, c\right)$ of Grassmann parity $\varepsilon\left(F_{3}\right)=\varepsilon$, which depends on the new positions $q^{i}$ and the old momenta $p_{j}$, such that

$$
-\mathrm{d} p_{i} q^{i}=p_{j}^{\prime} \mathrm{d} q^{\prime j}+\mathrm{d} F_{3}, \quad q^{i}=-\left(\frac{\overrightarrow{\partial^{\ell}}}{\partial p_{i}} F_{3}\right), \quad p_{j}^{\prime}=-\left(F_{3} \frac{\overleftarrow{\partial^{r}}}{\partial q^{\prime j}}\right)
$$

(The most general coordinate transformation in $\mathcal{G}_{1}$ is a finite composition of $F_{3}$ type transformations (5.1). This can for instance be proven with the help of Moser's trick [23].) The new momenta $p_{j}^{\prime}=p_{j}^{\prime}(p)$ should still be Casimirs for the second Poisson bracket,

$$
\begin{aligned}
0 & =\left\{p_{i}^{\prime}, c_{\tilde{k}}\right\}^{2}=\left(p_{i}^{\prime} \frac{\overleftarrow{\partial^{r}}}{\partial q^{\prime j}}\right)\left\{q^{\prime j}, c_{\tilde{k}}\right\}^{2}+\left(p_{i}^{\prime} \frac{\overleftarrow{\partial^{r}}}{\partial p_{j}}\right)\left\{p_{j}, c_{\tilde{k}}\right\}^{2}+\left(p_{i}^{\prime} \frac{\overleftarrow{\partial^{r}}}{\partial c_{\tilde{\jmath}}}\right)\left\{c_{\tilde{\jmath}}, c_{\tilde{k}}\right\}^{2} \\
& =-\left(F_{3} \frac{\overleftarrow{\partial^{r}}}{\partial q^{\prime i}} \frac{\overleftarrow{\partial^{r}}}{\partial q^{\prime j}}\right) E_{\tilde{k}}^{\prime j} .
\end{aligned}
$$


Since the new matrix $E_{\tilde{k}}^{\prime j}$ must be invertible, cf. Observation 2.6, the second derivatives of $F_{3}$ with respect to the $q^{\prime}$ variables must vanish,

$$
\left(F_{3} \frac{\overleftarrow{\partial^{r}}}{\partial q^{\prime i}} \frac{\overleftarrow{\partial^{r}}}{\partial q^{\prime j}}\right)=0
$$

Hence the generator

$$
-F_{3}=A_{j}(p, c) q^{j}+B(p, c)
$$

is affine in the new positions $q^{i}$. (The minus sign is introduced for later convenience. At this stage, the $F_{3}$ coefficient functions $A_{j}=A_{j}(p, c)$ and $B=B(p, c)$ are supposed to be independent of any previous definitions.) The new momenta $p_{j}^{\prime}$ become the $A_{j}$ coefficient functions,

$$
p_{j}^{\prime}(p)=-\left(F_{3} \frac{\overleftarrow{\partial^{r}}}{\partial q^{\prime j}}\right)=A_{j}(p, c)
$$

In particular, we conclude the following Observation 5.4.

Observation 5.4 The $F_{3}$ coefficient functions $A_{j}=A_{j}(p)$ must be independent of the c variables.

\subsection{Positions $q^{i}$}

The positions $q^{i} \rightarrow q^{\prime j}$ transform affinely under coordinate transformations $z^{A} \rightarrow z^{B}=z^{B}(z)$ in $\mathcal{G}$,

$$
q^{i}=-\left(\frac{\overrightarrow{\partial^{\ell}}}{\partial p_{i}} F_{3}\right)=\left(\frac{\overrightarrow{\partial^{\ell}}}{\partial p_{i}} A_{j}\right) q^{j}+\left(\frac{\overrightarrow{\partial^{\ell}}}{\partial p_{i}} B\right)
$$

Combined with transformations from $\mathcal{G}_{2}$, eq. (5.6) proves the following Proposition 5.5.

Proposition 5.5 (Affinity) The bundle $\mathcal{M} \rightarrow \mathcal{N}$ is an affine fiber bundle. Under a coordinate transformation $z^{A} \rightarrow z^{B}=z^{B}(z)$ that belongs to the groupoid $\mathcal{G}$, the positions $q^{i} \rightarrow q^{j}$ transform affinely with Jacobian matrix given by

$$
\left(q^{i} \frac{\overleftarrow{\partial^{r}}}{\partial q^{\prime j}}\right)=\left(\frac{\overrightarrow{\partial^{\ell}}}{\partial p_{i}} p_{j}^{\prime}\right) \quad \Leftrightarrow \quad\left(\frac{\overrightarrow{\partial^{\ell}}}{\partial q^{\prime j}} q^{i}\right)=(-1)^{\left(\varepsilon_{i}+\varepsilon_{j}\right)(1-\varepsilon)}\left(p_{j}^{\prime} \frac{\overleftarrow{\partial^{r}}}{\partial p_{i}}\right)
$$

\section{$5.4 E_{\tilde{\jmath}}^{i}$ matrix}

Returning again to just the $F_{3}$ transformation (5.1) from Subsection 5.2, the $E_{\tilde{k}}^{i}$ matrix (3.3) transforms $E_{\tilde{k}}^{i} \rightarrow E_{\tilde{k}}^{j}$ as a tensor

$$
\begin{aligned}
E_{\tilde{k}}^{i}:= & \left\{q^{i}, c_{\tilde{k}}\right\}^{2}=\left(q^{i} \frac{\overleftarrow{\partial^{r}}}{\partial q^{\prime j}}\right)\left\{q^{\prime j}, c_{\tilde{k}}\right\}^{2}+\left(q^{i} \frac{\overleftarrow{\partial^{r}}}{\partial p_{j}}\right)\left\{p_{j}, c_{\tilde{k}}\right\}^{2}+\left(q^{i} \frac{\overleftarrow{\partial^{r}}}{\partial c_{\tilde{\jmath}}}\right)\left\{c_{\tilde{\jmath}}, c_{\tilde{k}}\right\}^{2} \\
= & \left(\frac{\overrightarrow{\partial^{\ell}}}{\partial p_{i}} A_{j}\right) E_{\tilde{k}}^{\prime j}=\left(\frac{\partial^{\ell}}{\partial p_{i}} p_{j}^{\prime}\right) E_{\tilde{k}}^{\prime j} .
\end{aligned}
$$

Combined with transformations from $\mathcal{G}_{2}$, eq. (5.8) proves the following Proposition 5.6. 
Proposition 5.6 Under a coordinate transformation $z^{A} \rightarrow z^{\prime B}=z^{\prime B}(z)$ that belongs to the groupoid $\mathcal{G}$, the upper (lower) index of the $E^{i}{ }_{j}$ matrix (3.3) transforms as contravariant (covariant) tensor

$$
E_{\tilde{m}}^{i}=\left(\frac{\overrightarrow{\partial^{\ell}}}{\partial p_{i}} p_{j}^{\prime}\right) E_{\tilde{k}}^{\prime j}\left(\frac{\overrightarrow{\partial^{\ell}}}{\partial c_{\tilde{k}}^{\prime}} c_{\tilde{m}}\right)
$$

of the corresponding descended coordinate transformation $p_{i} \rightarrow p_{j}^{\prime}=p_{j}^{\prime}(p)\left(c_{\tilde{\imath}} \rightarrow c_{\tilde{\jmath}}^{\prime}=c_{\tilde{\jmath}}^{\prime}(c)\right.$ ) of the local product manifold $\mathcal{N}$, respectively.

\subsection{Para-Dolbeault Differentials}

Inspired by the $d^{a}$-differentials (4.1), we define two sets of para-Dolbeault differentials,

$$
\begin{gathered}
\partial^{a}:=\mathrm{d} p_{i}\left\{q^{i}, \xi^{I}\right\} \frac{\overrightarrow{\partial^{\ell}}}{\partial \xi^{I}}, \quad \mathrm{~d}=\mathrm{d} z^{A} \frac{\overrightarrow{\partial^{\ell}}}{\partial z^{A}}=\mathrm{d} q^{i} \frac{\overrightarrow{\partial^{\ell}}}{\partial q^{i}}+\partial^{1}+\widetilde{\partial}^{1}, \\
\partial^{1}:=\mathrm{d} p_{i} \frac{\partial^{\ell}}{\partial p_{i}}, \quad \widetilde{\partial}^{1}:=\mathrm{d} c_{\tilde{\jmath}} \frac{\partial^{\ell}}{\partial c_{\tilde{\jmath}}} \\
\partial^{2}:=\mathrm{d} p_{i} E^{i}{ }_{\tilde{\jmath}} \frac{\overrightarrow{\partial^{\ell}}}{\partial c_{\tilde{\jmath}}}, \quad \widetilde{\partial}^{2}:=\mathrm{d} c_{\tilde{\imath}} \widetilde{E}^{\tilde{r}}{ }_{j} \frac{\partial^{\ell}}{\partial p_{j}}
\end{gathered}
$$

The definitions (5.10)-(5.12) are invariant under local coordinate transformations $z^{A} \rightarrow z^{\prime B}=z^{\prime B}(z)$ in $\mathcal{G}$, cf. Proposition 5.5 and Proposition 5.6. Note that whereas the $d^{a}$-differentials (4.1) are Grassmannodd and have form-degree 0, the differentials (5.10)-(5.12) are Grassmann-even and have form-degree 1, cf. Subsection 1.1. The $2 \times 2=4$ para-Dolbeault differentials $\partial^{a}$ and $\widetilde{\partial}^{b}$ satisfy

$$
\left[\partial^{a}, \partial^{b}\right]=0, \quad\left[\widetilde{\partial}^{a}, \widetilde{\partial}^{b}\right]=0, \quad\left[\partial^{a}, \widetilde{\partial}^{b}\right] \propto \epsilon^{a b},
$$

because of closedness conditions (4.11) and (4.15).

\subsection{Presymplectic Potential $\vartheta$}

Definition 5.7 The subgroupoid $\mathcal{G}_{0} \subseteq \mathcal{G}$ of restricted coordinate transformations consists of local coordinate transformations $z^{A} \rightarrow z^{B}=z^{B}(z)$ such that the positions $q^{i} \rightarrow q^{\prime j}$ transform linearly without an inhomogeneous term.

Definition 5.8 The subgroupoid $\mathcal{G}_{\text {gauge }} \subseteq \mathcal{G}$ of gauge transformations consists of local coordinate transformations $z^{A} \rightarrow z^{\prime B}=z^{\prime B}(z)$ that do not transform $p_{j}^{\prime}=p_{j}$ nor $c_{\tilde{k}}^{\prime}=c_{\tilde{k}}$ but do only transform the positions

$$
q^{i} \longrightarrow q^{i}=q^{i}-\left(\frac{\overrightarrow{\partial^{\ell}}}{\partial p_{i}} B\right)
$$

by an Abelian gauge transformation, where $B=B(p, c)$ is the gauge parameter.

Every coordinate transformations $z^{A} \rightarrow z^{\prime B}=z^{\prime B}(z)$ in $\mathcal{G}$ may be written as a composition of a restricted coordinate transformation and a gauge transformation from Definitions 5.7-5.8. 
Definition 5.9 The presymplectic potential $\vartheta$ is defined locally as

$$
\vartheta:=\mathrm{d} z^{A} \vartheta_{A}=\mathrm{d} q^{i} \vartheta_{i}+\mathrm{d} p_{j} \vartheta^{j}+\mathrm{d} c_{\tilde{k}} \vartheta^{\tilde{k}}:=-\mathrm{d} p_{j} q^{j},
$$

with components

$$
\vartheta_{i}:=0, \quad \vartheta^{j}:=-q^{j}, \quad \vartheta^{\tilde{k}}:=0, \quad \varepsilon(\vartheta)=\varepsilon
$$

In other words, the presymplectic potential $\vartheta$ is basically a gadget to keep track of the fiber coordinates $q^{i}$. The presymplectic potential $\vartheta$ itself is parallel to the $\mathcal{N}_{1}$ leaves, i.e., the restricted one-form $\vartheta$ has grading $(1,0)$ with respect to the first para-Dolbeault pair $\left(\partial^{1}, \widetilde{\partial}^{1}\right)$.

Proposition 5.10 The locally defined presymplectic potential $\vartheta$

1. behaves as a one-form (=co-vector) $\vartheta^{i}=\left(\frac{\overrightarrow{\partial^{\ell}}}{\partial p_{i}} p_{j}^{\prime}\right) \vartheta^{\prime j}$ under restricted coordinate transformations, with bi-grading $(1,0)$ with respect to the first para-Dolbeault pair $\left(\partial^{1}, \widetilde{\partial}^{1}\right)$;

2. and $\vartheta$ behaves as gauge potential

$$
\vartheta \longrightarrow \vartheta^{\prime}=\vartheta+\left(\partial^{1} B\right) \quad \Leftrightarrow \quad \vartheta^{i} \longrightarrow \vartheta^{\prime i}=\vartheta^{i}+\left(\frac{\overrightarrow{\partial^{\ell}}}{\partial p_{i}} B\right),
$$

under gauge transformations.

Proposition 5.10 shows that the fiber bundle $\mathcal{M} \rightarrow \mathcal{N}$ has a locally defined gauge potential/connection $\vartheta$, and a globally defined field strength/curvature

$$
\omega:=\mathrm{d} \vartheta=\mathrm{d} p_{i} \wedge \mathrm{d} q^{i} \in \Gamma\left(\bigwedge^{2}\left(T^{*} \mathcal{M}\right)\right),
$$

see Subsection 5.8. In particular, the gauge bundle is never flat. The presymplectic two-form $\omega$ is invariant under coordinate transformations $z^{A} \rightarrow z^{B}=z^{B}(z)$ in $\mathcal{G}$. It corresponds to the $\mathcal{M}_{1}$ foliations of symplectic leaves for the first Poisson bracket $\{\cdot, \cdot\}^{1}$.

\section{$5.7 \quad F^{i j}$ matrix}

Returning one more time to just the $F_{3}$ transformation (5.1) from Subsection 5.2, the $F^{i m}$ matrix (3.3) transforms $F^{i m} \rightarrow F^{\prime j k}$ with an inhomogeneous term

$$
\begin{aligned}
F^{i m} & -\left(\frac{\overrightarrow{\partial^{\ell}}}{\partial p_{i}} p_{j}^{\prime}\right) F^{\prime j k}\left(p_{k}^{\prime} \frac{\overleftarrow{\partial^{r}}}{\partial p_{m}}\right)(-1)^{\left(\varepsilon_{k}+\varepsilon_{m}\right)(1-\varepsilon)} \\
& =\left\{q^{i}, q^{m}\right\}^{2}-\left(q^{i} \frac{\partial^{r}}{\partial q^{\prime j}}\right)\left\{q^{\prime j}, q^{\prime k}\right\}^{2}\left(\frac{\overrightarrow{\partial^{\ell}}}{\partial q^{\prime k}} q^{m}\right) \\
& =\left(q^{i} \frac{\overleftarrow{\partial^{r}}}{\partial q^{\prime j}}\right)\left\{q^{\prime j}, c_{\tilde{k}}\right\}^{2}\left(\frac{\partial^{\ell}}{\partial c_{\tilde{k}}} q^{m}\right)+\left(q^{i} \frac{\partial^{r}}{\partial c_{\tilde{\jmath}}}\right)\left\{c_{\tilde{\jmath}}, q^{\prime k}\right\}^{2}\left(\frac{\overrightarrow{\partial^{\ell}}}{\partial q^{\prime k}} q^{m}\right)
\end{aligned}
$$




$$
\begin{aligned}
= & E_{\tilde{k}}^{i} \frac{\overrightarrow{\partial^{\ell}}}{\partial c_{\tilde{k}}}\left\{q^{m}, B\right\}^{1}-(-1)^{\varepsilon\left(p_{i}\right) \varepsilon\left(p_{m}\right)}(i \leftrightarrow m) \\
= & \left\{q^{i}, q^{k}\right\}^{2} \frac{\partial^{\ell}}{\partial q^{k}}\left\{q^{m}, B\right\}^{1}+\left\{q^{i}, p_{k}\right\}^{2} \frac{\overrightarrow{\partial^{\ell}}}{\partial p_{k}}\left\{q^{m}, B\right\}^{1} \\
& +\left\{q^{i}, c_{\tilde{k}}\right\}^{2} \frac{\overrightarrow{\partial^{\ell}}}{\partial c_{\tilde{k}}}\left\{q^{m}, B\right\}^{1}-(-1)^{\varepsilon\left(p_{i}\right) \varepsilon\left(p_{m}\right)}(i \leftrightarrow m) \\
= & \left\{q^{i},\left\{q^{m}, B\right\}^{1}\right\}^{2}-(-1)^{\varepsilon\left(p_{i}\right) \varepsilon\left(p_{m}\right)}(i \leftrightarrow m) .
\end{aligned}
$$

Proposition 5.11 The locally defined object

$$
F:=-\frac{1}{2} \mathrm{~d} p_{j} \wedge \mathrm{d} p_{i} F^{i j}=\frac{1}{2} \mathrm{~d} p_{i} F^{i j} \wedge \mathrm{d} p_{j}(-1)^{\varepsilon_{j}(1-\varepsilon)}, \quad F^{i j}=F^{i j}(p, c),
$$

which is formed from the $F^{i j}$ matrix (3.3),

1. behaves as a two-form $F^{i m}=\left(\frac{\overrightarrow{\partial^{\ell}}}{\partial p_{i}} p_{j}^{\prime}\right) F^{\prime j k}\left(p_{k}^{\prime} \overleftarrow{\frac{\partial^{r}}{\partial p_{m}}}\right)(-1)^{\left(\varepsilon_{k}+\varepsilon_{m}\right)(1-\varepsilon)}$ under restricted coordinate transformations, with bi-grading $(2,0)$ with respect to the first para-Dolbeault pair $\left(\partial^{1}, \widetilde{\partial}^{1}\right)$;

2. and $F$ behaves as

$$
\begin{aligned}
F & \underset{\Uparrow}{\mathbb{1}} \\
& F^{\prime}=F-\left(\partial^{2} \partial^{1} B\right)=F+\left(\partial^{1} \partial^{2} B\right) \\
F^{i j} & \longrightarrow F^{\prime i j}=F^{i j}-\left(E_{\tilde{k}}^{i}\left(\frac{\overrightarrow{\partial^{\ell}}}{\partial c_{\tilde{k}}} \frac{\partial^{\ell}}{\partial p_{j}} B\right)-(-1)^{\varepsilon\left(p_{i}\right) \varepsilon\left(p_{j}\right)}(i \leftrightarrow j)\right),
\end{aligned}
$$

under gauge transformations.

The restricted two-form $F$ from eq. (5.20) corresponds to the "two-form" $\beta^{2}$ from eq. (4.4). The $d^{a}$-closedness condition (4.10) for the two-form $\beta^{2}$ translates into that the two-form $F$ is $\partial^{a}$-closed,

$$
\left(\partial^{a} F\right)=0 \text {. }
$$

\subsection{Gauge Bundle}

We now rephrase the fiber bundle construction using the language of gauge bundles.

1. From the perspective of a gauge bundle over $\mathcal{N}$, the groupoid $\mathcal{G}_{0}$ of restricted coordinate transformations become by definition the only allowed coordinate transformations. Then the fiber bundle $\mathcal{M} \rightarrow \mathcal{N}$ becomes a linear vector bundle; and $\vartheta_{(\alpha)} \in \Gamma\left(\left.T^{*} \mathcal{M}\right|_{\mathbb{R}^{n} \times \mathcal{U}_{(\alpha)}}\right)$ and $F_{(\alpha)} \in \Gamma\left(\left.\bigwedge^{2}\left(T^{*} \mathcal{N}\right)\right|_{\mathcal{U}_{(\alpha)}}\right)$ become two families of differential forms, which are labeled by local neighborhoods $\mathcal{U}_{(\alpha)} \subseteq \mathcal{N}$.

2. It should be stressed that the word gauge bundle in this paper is used in a slightly non-standard way. Although $\vartheta_{(\alpha)}$ plays the rôle of a gauge potential/connection, it is not an ordinary gauge potential, since besides dependence on the base coordinates $\xi_{(\alpha)}^{I}$, it also depends on the fiber coordinates $q_{(\alpha)}^{i}$. Another peculiarity is that a change of the base coordinates $p_{(\alpha) i} \rightarrow p_{(\alpha) j}^{\prime}=$ $p_{(\alpha) j}^{\prime}\left(p_{(\alpha) j}\right)$ induces a corresponding change in the fiber coordinates $q_{(\alpha)}^{i}=\left(\frac{\overrightarrow{\partial^{\ell}}}{\partial p_{(\alpha) i}} p_{(\alpha) j}^{\prime}\right) q_{(\alpha)}^{\prime j}$, cf. Proposition 5.5. 
3. A gauge transformation from an $(\alpha)$-gauge in a local patch $\mathcal{U}_{(\alpha)}$ to a $(\beta)$-gauge in a local patch $\mathcal{U}_{(\beta)}$ makes sense if the overlap $\mathcal{U}_{(\alpha)} \cap \mathcal{U}_{(\beta)} \neq \emptyset$ is non-empty. The gauge transformation is

$$
q_{(\beta)}^{i}=q_{(\alpha)}^{i}-\left(\frac{\partial^{\ell}}{\partial p_{i}} B_{(\alpha \beta)}\right), \quad \vartheta_{(\beta)}=\vartheta_{(\alpha)}+\left(\partial^{1} B_{(\alpha \beta)}\right), \quad F_{(\beta)}=F_{(\alpha)}+\left(\partial^{1} \partial^{2} B_{(\alpha \beta)}\right),
$$

with gauge parameter $B_{(\alpha \beta)}=B_{(\alpha \beta)}(\xi)$.

4. For a triple overlap $\mathcal{U}_{(\alpha)} \cap \mathcal{U}_{(\beta)} \cap \mathcal{U}_{(\gamma)} \neq \emptyset$, one must demand the cocycle condition

$$
B_{(\alpha \beta)}+B_{(\beta \gamma)}+B_{(\gamma \alpha)}=C_{(\alpha \beta \gamma)}
$$

for some functions $C_{(\alpha \beta \gamma)}$ with $\left(\partial^{1} C_{(\alpha \beta \gamma)}\right)=0$, i.e., functions $C_{(\alpha \beta \gamma)}=C_{(\alpha \beta \gamma)}(c)$ that only depend on the $c$ coordinates.

\section{Proof of Bi-Darboux Theorem}

\subsection{Factorization Condition}

We next continue with the proof of bi-Darboux Theorem 3.2. Note that Proposition 5.6 shows immediately that the factorization condition (3.5) is necessary for the bi-Darboux Theorem 3.2.

On the other hand, let us from now on assume that the $E=P C$ factorization condition (3.5) is satisfied. It then follows from the two closedness conditions (4.11) and (4.15) that the $P$ and $C$ matrix factors are Jacobi matrices, i.e., there exist locally some reparametrizations $p_{i} \rightarrow p_{j}^{\prime}=p_{j}^{\prime}(p)$ and $c_{\tilde{\imath}} \rightarrow c_{\tilde{\jmath}}^{\prime}=c_{\tilde{\jmath}}^{\prime}(c)$, such that

$$
P_{j}^{i}=\left(\frac{\overrightarrow{\partial^{\ell}}}{\partial p_{i}} p_{j}^{\prime}\right), \quad\left(C^{-1}\right)^{\tilde{\imath}}{ }_{\tilde{\jmath}}=\left(\frac{\overrightarrow{\partial^{\ell}}}{\partial c_{\tilde{\imath}}} c_{\tilde{\jmath}}^{\prime}\right),
$$

respectively. Thus by choosing the $F_{3}$ coefficient functions $A_{j}$ in eq. (5.4) to be the new $p_{j}^{\prime}$ variables (6.1) (and letting the $B$ function in eq. (5.4) be arbitrary, e.g., zero), it is possible to perform a $F_{3}$ transformation in $\mathcal{G}_{1}$, and a reparametrization $c_{\tilde{\imath}} \rightarrow c_{\tilde{\jmath}}^{\prime}=c_{\tilde{\jmath}}^{\prime}(c)$ in $\mathcal{G}_{2}$, such that the new $E^{\prime j}$ matrix (3.3) becomes the unit matrix $E_{\tilde{k}}^{j}=\delta_{\tilde{k}}^{j}$.

It still remains to show that the new $F^{\prime j k}$ matrix (3.3) can be chosen to be zero $F^{\prime j k}=0$. This will be done in the next Subsection 6.2 with the help of bi-Poincaré Lemma A.1.

\subsection{Bi-Poincaré Lemma}

Let us from now on assume that the $E^{i}$ matrix (3.3) is the unit matrix $E^{i}{ }_{\tilde{j}}=\delta_{\tilde{\jmath}}^{i}$. Now recall the two $d^{a}$ differentials (4.2) and the $d^{a}$-closedness condition (4.10) for the two-form $\beta^{2}$ in Section 4 . Treating the $q^{i}$ variables as passive spectator parameters, we are now in the position to apply the bi-Poincaré Lemma A.1 with the triple $\left\{p_{i} ; c_{\tilde{\jmath}} ; \eta_{k}\right\}$ as active variables $\left\{x_{1}^{i} ; x_{2}^{\tilde{j}} ; x_{3}^{k}\right\}$. There hence exists a zero-form $B=B(p, c) \in \mathcal{F}$, of Grassmann parity $\varepsilon(B)=\varepsilon$, such that

$$
\beta^{2}=d^{2} d^{1} B=\eta_{i}\left\{q^{i},\left\{q^{j}, B\right\}^{1}\right\}^{2} \eta_{j}(-1)^{\varepsilon\left(\eta_{j}\right) \varepsilon},
$$

or explicitly,

$$
F^{i j}:=\left\{q^{i}, q^{j}\right\}^{2}=\left\{q^{i},\left\{q^{j}, B\right\}^{1}\right\}^{2}-(-1)^{\left(\varepsilon_{i}+\varepsilon\right)\left(\varepsilon_{j}+\varepsilon\right)}(i \leftrightarrow j)
$$


By shifting the $q$ variables as

$$
q^{i} \quad \longrightarrow \quad q^{i}=q^{i}-\left\{q^{i}, B\right\}^{1}=q^{i}-\left(\frac{\overrightarrow{\partial^{\ell}}}{\partial p_{i}} B\right)
$$

we achieve that the matrix

$$
F^{i j}:=\left\{q^{i}, q^{j}\right\}^{2} \quad \longrightarrow \quad F^{\prime i j}:=\left\{q^{i}, q^{\prime j}\right\}^{2}=0
$$

vanishes, while all the other fundamental Poisson brackets $\left\{z^{A}, z^{B}\right\}^{a}$ remain unchanged. Or equivalently, we may note by comparing eqs. (5.19) and (6.3) that the canonical transformation

$$
-F_{3}=p_{j} q^{j}+B
$$

leads to $F^{\prime j k}=0$. We have thus achieved a canonical form for the second Poisson bracket, and thereby confirmed that the factorization condition (3.5) is sufficient for the bi-Darboux Theorem 3.2.

\section{Bi-Canonical Transformations}

Let there be given a $3 n$-dimensional triplectic manifold $\left(\mathcal{M} ;\{\cdot, \cdot\}^{a}\right)$.

Definition 7.1 A bi-canonical transformation is a coordinate transformation $\left\{z^{A}\right\}=\left\{q^{i} ; p_{a j}\right\} \longrightarrow$ $\left\{z^{\prime A}\right\}=\left\{q^{i} ; p_{a j}^{\prime}\right\}$ between two bi-Darboux coordinate systems (2.7) of positions and momenta.

Proposition 7.2 Necessary conditions for a bi-canonical transformation $z^{A} \rightarrow z^{B}=z^{\prime B}(z)$ are the following.

1. The momenta $p_{a i}$ (i.e., the Casimirs $\left.\xi_{a i}\right)$ transform under rigid affine reparametrizations $p_{a i} \rightarrow$ $p_{a j}^{\prime}=p_{a j}^{\prime}\left(p_{a}\right)$ for each $a \in\{1,2\}$, with common constant $n \times n$ Jacobi matrix

$$
J_{j}^{i}:=\left(\frac{\overrightarrow{\partial^{\ell}}}{\partial p_{a i}} p_{a j}^{\prime}\right),
$$

(no sum over a). In particular, the Jacobi matrix $J^{i}{ }_{j}$ must be independent of a $\in\{1,2\}$.

2. The transformation of the position coordinates $q^{i}=J^{i}{ }_{j} q^{j}+b^{i}$ is composed of a rigid constant rotation with the Jacobi matrix (7.1) plus a shift $b^{i}=b^{i}(p)$.

Given a bi-canonical transformation $z \rightarrow z^{\prime}$, one can locally always perform an additional restricted bi-canonical transformation, $z^{\prime} \rightarrow z^{\prime \prime}$

$$
q^{\prime \prime i}=J^{i}{ }_{j} q^{\prime j}, \quad p_{a j}^{\prime}=p_{a i}^{\prime \prime} J_{j}^{i},
$$

involving the same constant Jacobi matrix (7.1), so that the combined bi-canonical transformation $z \rightarrow z^{\prime} \rightarrow z^{\prime \prime}$ is just a gauge transformation, cf. Definitions 5.7-5.8. The following Proposition 7.3 is a consequence of Proposition 5.11. 
Proposition 7.3 A necessary and sufficient condition for a bi-canonical gauge transformation $q^{i} \rightarrow$ $q^{i}=q^{i}-b^{i}$ is that locally the shift $b^{i}=b^{i}(p)$ is a gradient with respect to both sets of momenta, i.e., there locally exist $B^{a}=B^{a}(p), a \in\{1,2\}$, such that

$$
b^{i}=\left(\frac{\overrightarrow{\partial^{\ell}}}{\partial p_{a i}} B^{a}\right)
$$

(no sum over a).

The main lesson is that bi-canonical transformations are rigid, in contrast to standard canonical transformations, which figuratively speaking, exhibit flexible behavior, which is capable of washing out local features.

\section{Para-Hypercomplex Structure}

\subsection{Almost Parity Structures}

An almost parity structure $P: \Gamma(T \mathcal{N}) \rightarrow \Gamma(T \mathcal{N})$,

$$
P=\partial_{I}^{r} P_{J}^{I} \otimes \overrightarrow{\mathrm{d}}^{J}, \quad \varepsilon\left(P_{J}^{I}\right)=\varepsilon_{I}+\varepsilon_{J}
$$

(also known as an almost para-complex structure or an almost local product structure) is a (mixed contravariant and covariant) tensor $P^{I}{ }_{J}$ that satisfies [24]

$$
P^{2}=\mathrm{Id}=\partial_{I}^{r} \otimes \overrightarrow{\mathrm{d} \xi^{I}}, \quad \varepsilon(P)=0, \quad \operatorname{dim} \operatorname{ker}(P \pm \mathrm{Id})=n \equiv \frac{1}{2} \operatorname{dim}(\mathcal{N}) .
$$

Here $\partial_{I}^{r} \equiv(-1)^{\varepsilon_{I}} \partial_{I}^{\ell}$ are not usual partial derivatives. In particular, they do not act on the tensor $P^{I}{ }_{J}$ in eq. (8.1). Rather they are a dual basis to the one-forms $\overrightarrow{\mathrm{d}}^{I}$ :

$$
\overrightarrow{\mathrm{d} x^{I}}\left(\partial_{J}^{r}\right)=\delta_{J}^{I} .
$$

Phrased differently, the $\partial_{I}^{r}$ are merely bookkeeping devices, that transform as right partial derivatives under general coordinate transformations. (To be able to distinguish them from true partial derivatives, the differentiation variable $\xi^{I}$ on a true partial derivative $\partial / \partial \xi^{I}$ is written explicitly.) It is convenient to introduce two idempotent projection operators

$$
P_{ \pm}:=\frac{1}{2}(\operatorname{Id} \pm P), \quad \operatorname{Id}=P_{+}+P_{-}, \quad P=P_{+}-P_{-}, \quad P_{ \pm} P_{ \pm}=P_{ \pm}, \quad P_{ \pm} P_{\mp}=0 .
$$

\subsection{Parity Structures}

We start by defining two chiral Nijenhuis tensors $N_{ \pm}: \Gamma(T \mathcal{N}) \times \Gamma(T \mathcal{N}) \rightarrow \Gamma(T \mathcal{N})$,

$$
N_{ \pm}(X, Y):=P_{\mp}\left[P_{ \pm} X, P_{ \pm} Y\right]=-(-1)^{\varepsilon(X) \varepsilon(Y)} N_{ \pm}(Y, X)
$$

where $X, Y \in \Gamma(T \mathcal{N})$ are vector fields. Note that

$$
N_{ \pm}(X, P Y)= \pm N_{ \pm}(X, Y)=N_{ \pm}(P X, Y) .
$$


The Nijenhuis tensor $N=\frac{1}{2} \partial_{K}^{r} N_{I J}^{K} \otimes \overrightarrow{\mathrm{d}}^{J} \wedge \overrightarrow{\mathrm{d}}^{I} \in \Gamma\left(T \mathcal{N} \otimes \bigwedge^{2}\left(T^{*} \mathcal{N}\right)\right)$ is defined as

$$
N:=4\left(N_{+}+N_{-}\right),
$$

or

$$
N(X, Y)=[X, Y]+[P X, P Y]-P[X, P Y]-P[P X, Y]=-(-1)^{\varepsilon(X) \varepsilon(Y)} N(Y, X) .
$$

Equivalently in components,

$$
-\overrightarrow{\mathrm{d}}^{K}\left(N\left(\partial_{I}^{r}, \partial_{J}^{r}\right)\right)=N_{I J}^{K}=\left(\left(P^{K}{ }_{I} \frac{\overleftarrow{\partial^{r}}}{\partial \xi^{M}}\right) P_{J}^{M}-P_{M}^{K}\left(P^{M}{ }_{I} \frac{\overleftarrow{\partial^{r}}}{\partial \xi^{J}}\right)\right)-(-1)^{\varepsilon_{I} \varepsilon_{J}}(I \leftrightarrow J)
$$

The relation can be inverted to give

$$
\begin{aligned}
8 N_{ \pm}(X, Y) & =N(X, Y) \pm N(X, P Y)=2 N\left(X, P_{ \pm} Y\right) \\
8 N_{ \pm I J}^{K} & =N^{K}{ }_{I M} P_{ \pm J}^{M}-(-1)^{\varepsilon_{I} \varepsilon_{J}}(I \leftrightarrow J)
\end{aligned}
$$

Definition 8.1 An almost parity structure $P: \Gamma(T \mathcal{N}) \rightarrow \Gamma(T \mathcal{N})$ becomes a parity structure if the two chiral Nijenhuis tensors $N_{ \pm}=0$ vanish.

One may show that the two chiral Nijenhuis tensors $N_{ \pm}=0$ vanish iff the corresponding Nijenhuis tensor $N=0$ vanishes. The existence of a parity structure $P: \Gamma(T \mathcal{N}) \rightarrow \Gamma(T \mathcal{N})$ implies that $P_{ \pm}(T \mathcal{N}) \subseteq T \mathcal{N}$ are two integrable distributions, and that the holonomy of the manifold $\mathcal{N}$ is $\subseteq$ $G L(n) \times G L(n)$.

\subsubsection{Parity Structure $\Sigma$}

Recall from Section 2.6 that the base manifold $\mathcal{N}$ is a local product manifold with local coordinates

$$
\xi^{I}=\left[\begin{array}{c}
p_{i} \\
c_{\tilde{\imath}}
\end{array}\right], \quad \partial_{I}^{\ell}=\left[\begin{array}{c}
\partial_{\ell}^{i} \\
\partial_{\ell}^{\tilde{i}}
\end{array}\right]
$$

An obvious choice of parity structure, which we will call $\Sigma: \Gamma(T \mathcal{N}) \rightarrow \Gamma(T \mathcal{N})$, preserves (inverts) all the tangent directions $\subseteq T \mathcal{N}$ of the $\mathcal{N}_{1}$ leaves $\left(\mathcal{N}_{2}\right.$ leaves) (2.13), respectively,

$$
\begin{aligned}
& \Sigma:=\Sigma_{+}-\Sigma_{-}, \quad \Sigma_{+}:=\partial_{r}^{i} \otimes \overrightarrow{\mathrm{d} p_{i}}, \quad \Sigma_{-}:=\partial_{r}^{\tilde{\imath}} \otimes \overrightarrow{\mathrm{d} c_{\tilde{\imath}}} \\
& \Sigma_{J}^{I}:=\left[\begin{array}{cc}
c_{i}^{j} & 0 \\
0 & -\delta_{\tilde{\imath}}^{\tilde{\jmath}}
\end{array}\right], \quad \Sigma\left(\partial_{\ell}^{i}\right):=\partial_{\ell}^{i}, \quad \Sigma\left(\partial_{\ell}^{\tilde{\jmath}}\right):=-\partial_{\ell}^{\tilde{\jmath}} .
\end{aligned}
$$

The matrix $\Sigma_{J}^{I}$ behaves a mixed tensor under coordinate transformations of $\mathcal{N}$. The first pair (5.11) of para-Dolbeault differentials satisfies

$$
\partial^{1}=\Sigma_{+}^{T} \mathrm{~d}, \quad \widetilde{\partial}^{1}=\Sigma_{-}^{T} \mathrm{~d}, \quad \Sigma_{ \pm}:=\frac{1}{2}(\operatorname{Id} \pm \Sigma) .
$$

Here $\Sigma_{+}^{T}: \Gamma\left(T^{*} \mathcal{N}\right) \rightarrow \Gamma\left(T^{*} \mathcal{N}\right)$ and $\Sigma_{-}^{T}: \Gamma\left(T^{*} \mathcal{N}\right) \rightarrow \Gamma\left(T^{*} \mathcal{N}\right)$ are the projection operators to the $\mathcal{N}_{1}$ and $\mathcal{N}_{2}$ leaf directions, respectively; see also eqs. (8.4) and (8.19). 


\subsubsection{Parity Structure $P$}

The invertible $E^{i}{ }_{\tilde{\jmath}}$ matrix (3.3) yields another parity structure

$$
\begin{gathered}
P:=\partial_{r}^{i} \widetilde{E}_{i}^{\tilde{\jmath}} \otimes \overrightarrow{\mathrm{d}}_{\tilde{\jmath}}+\partial_{r}^{\tilde{\imath}} E_{\tilde{\imath}}^{j} \otimes \overrightarrow{\mathrm{d}}_{j}, \\
P_{J}^{I}:=\left[\begin{array}{cc}
0 & \widetilde{E}_{i}^{\tilde{j}} \\
E_{\tilde{\imath}}{ }^{j} & 0
\end{array}\right], \quad P\left(\partial_{\ell}^{i}\right):=E_{\tilde{\jmath}}^{i} \partial_{\ell}^{\tilde{\jmath}}, \quad P\left(\partial_{\ell}^{\tilde{\jmath}}\right):=\widetilde{E}_{i}^{j} \partial_{\ell}^{i},
\end{gathered}
$$

where we have defined transposed matrices

$$
P_{I}^{J}:=(-1)^{\varepsilon_{I}\left(\varepsilon_{J}+1\right)} P_{I}^{J}, \quad E_{\tilde{\jmath}}^{i}:=(-1)^{\varepsilon\left(p_{i}\right)\left(\varepsilon\left(c_{\tilde{\jmath}}\right)+1\right)} E_{\tilde{\jmath}}^{i}, \quad \widetilde{E}_{i}^{\tilde{\jmath}}:=(-1)^{\varepsilon\left(c_{\tilde{\jmath}}\right)\left(\varepsilon\left(p_{i}\right)+1\right)} \widetilde{E}_{i}^{\tilde{j}} .
$$

There is an equivalent transposed formulation $P^{T}: \Gamma\left(T^{*} \mathcal{N}\right) \rightarrow \Gamma\left(T^{*} \mathcal{N}\right)$ on the cotangent space,

$$
\begin{gathered}
P^{T}=\mathrm{d} \xi^{I} P_{I}^{J} \otimes \overrightarrow{i_{J}^{\ell}}=\mathrm{d} p_{i} E_{\tilde{\jmath}}^{i} \otimes \overrightarrow{i_{\ell}^{\tilde{\jmath}}}+\mathrm{d} c_{\tilde{\imath}} \widetilde{E}^{\tilde{\imath}}{ }_{j} \otimes \overrightarrow{i_{\ell}^{j}}, \quad \overrightarrow{i_{J}^{\ell}}\left(\mathrm{d} \xi^{I}\right)=\delta_{J}^{I}, \\
P_{I}^{J}=\left[\begin{array}{cc}
0 & E^{i}{ }_{\tilde{\jmath}} \\
\widetilde{E}^{\tilde{\imath}}{ }_{j} & 0
\end{array}\right], \quad P^{T}\left(\mathrm{~d} c_{\tilde{\jmath}}\right)=\mathrm{d} p_{i} E^{i}{ }_{\tilde{\jmath}}, \quad P^{T}\left(\mathrm{~d} p_{i}\right)=\mathrm{d} c_{\tilde{\jmath}} \widetilde{E}^{\tilde{\jmath}}{ }_{i} .
\end{gathered}
$$

The identity $P^{2}=$ Id follows because $\widetilde{E}:=E^{-1}$ is the inverse of the $E$ matrix (3.3). The vanishing of the corresponding Nijenhuis tensor $N=0$ follows from the integrability conditions eqs. (4.11) and (4.15). The second pair (5.12) of para-Dolbeault differentials satisfies

$$
\partial^{2}=P^{T} \widetilde{\partial}^{1}, \quad \widetilde{\partial}^{2}=P^{T} \partial^{1} .
$$

\subsection{Para-Hypercomplex Structure}

The two parity structures $\Sigma$ and $P$ from Subsections 8.2.1-8.2.2 anticommute

$$
\{\Sigma, P\}_{+}:=\Sigma P+P \Sigma=0 \text {. }
$$

Conversely, any parity structure may be locally diagonalized to the form of $\Sigma: T \mathcal{N} \rightarrow T \mathcal{N}$ given in Subsection 8.2.1. This is just rephrasing the fact that a manifold equipped with a parity structure is the same as a local product manifold, cf. Subsection 2.6. Moreover, it is easy to see that any second parity structure $P: T \mathcal{N} \rightarrow T \mathcal{N}$ that anticommute eq. (8.22) must then be of the form given in Subsection 8.2.2 for some matrix $E_{j}^{i}$ that satisfies integrability conditions (4.11) and (4.15).

We may then define a complex structure as

$$
J:=P \Sigma, \quad J^{2}=-\mathrm{Id} .
$$

Together $\{\Sigma ; P ; J\}$ span a para-hypercomplex structure. See also Subsection 8.5.

Theorem 8.2 A triplectic fiber bundle $\left(\mathcal{M} \rightarrow \mathcal{N} ;\{\cdot, \cdot\}^{a}\right)$ is a para-hypercomplex gauge bundle with a $\partial^{a}$-closed (2,0)-form $F$. Conversely, for a given para-hypercomplex gauge bundle $\mathcal{M} \rightarrow \mathcal{N}$ with a $\partial^{a}$ closed $(2,0)$-form $F$, the total space $\mathcal{M}$ may be endowed with a triplectic structure $\{\cdot, \cdot\}^{a}, a \in\{1,2\}$. 
Here the $(2,0)$ bi-grading refers to the first para-Dolbeault pair $\left(\partial^{1}, \widetilde{\partial}^{1}\right)$. The one-to-one correspondence in Theorem 8.2 holds, basically because all possible consequences of the symmetrized Jacobi identity (2.3) have been completely transcribed into the gauge bundle language, cf. Subsection 5.8. Note that the dimension of a para-hypercomplex manifold $\mathcal{N}$ must be a multiplum of 2 (unlike a hypercomplex manifold, whose dimension must always be a multiplum of 4.)

A 2-dimensional example. Let the manifold be $\mathcal{N}=\mathbb{C}=\mathbb{R}^{2}$ with global coordinates $\left\{\xi^{I}\right\}=\left\{p_{1} ; c_{1}\right\}$. Let

$$
\Sigma=\left[\begin{array}{cc}
1 & 0 \\
0 & -1
\end{array}\right], \quad P=\left[\begin{array}{ll}
0 & 1 \\
1 & 0
\end{array}\right], \quad J=\left[\begin{array}{cc}
0 & -1 \\
1 & 0
\end{array}\right] .
$$

Let the non-zero fundamental Poisson brackets be $\left\{q^{1}, p_{1}\right\}^{1}=1=\left\{q^{1}, c_{1}\right\}^{2}$.

\subsection{The Obata Connection $\nabla$}

Proposition 8.3 (Superversion of the Obata connection [14]) There exists a unique torsionfree connection $\nabla: \Gamma(T \mathcal{N}) \times \Gamma(T \mathcal{N}) \rightarrow \Gamma(T \mathcal{N})$, that preserves the two anticommuting parity structures $\Sigma$ and $P$, i.e.,

$$
\nabla \Sigma=0, \quad \nabla P=0
$$

PROOF: The second condition in eq. (8.25) reads in components

$$
0=\left(\nabla_{I}^{\ell} P\right)^{J}{ }_{K}=\left(\frac{\overrightarrow{\partial^{\ell}}}{\partial \xi^{I}} P^{J}{ }_{K}\right)+\Gamma_{I}{ }^{J}{ }_{M} P^{M}{ }_{K}-(-1)^{\varepsilon_{I} \varepsilon_{J}} P^{J}{ }_{M} \Gamma^{M}{ }_{I K},
$$

where by definition

$$
\Gamma_{I K}^{J}=(-1)^{\varepsilon_{I} \varepsilon_{J}} \Gamma_{I K}^{J} \text {. }
$$

The torsion-free condition $T=0$ means that the Christoffel symbols are graded symmetric in the lower indices

$$
\Gamma_{I J}^{K}=-(-1)^{\left(\varepsilon_{I}+1\right)\left(\varepsilon_{J}+1\right)} \Gamma_{J I}^{K} .
$$

We may take $\Sigma$ and $P$ as in Subsections 8.2.1-8.2.2. The first condition in eq. (8.25) shows that all the mixed components of the Christoffel symbols $\Gamma^{K}{ }_{I J}$ vanish. The remaining two non-mixed components can be deduced of from eq. (8.26).

$$
\begin{aligned}
& -\Gamma_{j}^{i k}=\left(\frac{\overrightarrow{\partial^{\ell}}}{\partial p_{i}} \widetilde{E}_{j}^{\tilde{m}}\right) E_{\tilde{m}}^{k}=E_{\tilde{m}}^{i}\left(\widetilde{E}^{\tilde{m}}{ }_{j} \frac{\overleftarrow{\partial^{r}}}{\partial p_{k}}\right), \quad(-1)^{\varepsilon\left(p_{i}\right)} \Gamma_{k}^{i j}=\widetilde{E}_{k}^{\tilde{m}}\left(E_{\tilde{m}}^{i} \frac{\overleftarrow{\partial^{r}}}{\partial p_{j}}\right), \\
& -\Gamma_{\tilde{\jmath}}^{\tilde{\imath} \tilde{k}}=\left(\frac{\partial^{\ell}}{\partial c_{\tilde{\imath}}} E_{\tilde{\jmath}}{ }^{m}\right) \widetilde{E}_{m}^{\tilde{k}}=\widetilde{E}_{m}^{\tilde{\imath}}\left(E^{m}{ }_{\tilde{\jmath}} \frac{\overleftarrow{\partial^{r}}}{\partial c_{\tilde{k}}}\right), \quad(-1)^{\varepsilon\left(c_{\tilde{\imath}}\right)} \Gamma_{\tilde{k}}^{\tilde{\tilde{j}} \tilde{\jmath}}=E_{\tilde{k}}^{m}\left(\widetilde{E}_{m}{ }^{\tilde{\imath}} \frac{\overleftarrow{\partial^{r}}}{\partial c_{\tilde{\jmath}}}\right) .
\end{aligned}
$$

Theorem 8.4 The Obata connection $\nabla$ is flat if and only if the factorization condition (3.5) holds ${ }^{\ddagger \ddagger}$.

In other words, a triplectic fiber bundle $\left(\mathcal{M} \rightarrow \mathcal{N} ;\{\cdot, \cdot\}^{a}\right)$ has locally bi-Darboux coordinates iff the Obata connection $\nabla$ on the para-hypercomplex manifold $\mathcal{N}$ is flat.

\footnotetext{
$\ddagger^{\ddagger}$ Theorem 8.4 is essentially stated as Proposition 4.2 in Ref. [13]
} 


\subsection{Global $G L(2)$ Covariance}

Recall that the $g l(2)$ Lie algebra generators

$$
t_{0}=\left[\begin{array}{ll}
1 & 0 \\
0 & 1
\end{array}\right], \quad t_{1}=\left[\begin{array}{ll}
0 & 1 \\
1 & 0
\end{array}\right], \quad t_{2}=\left[\begin{array}{cc}
0 & -1 \\
1 & 0
\end{array}\right], \quad t_{3}=\left[\begin{array}{cc}
1 & 0 \\
0 & -1
\end{array}\right],
$$

form the algebra (B.12) of para-quaternions, also known as the algebra of split quaternions.

Observation 8.5 The $g l(2)$-generators $t_{\alpha}, \alpha \in\{0,1,2,3\}$, yields a representation of $\mathrm{Id}, P, J$ and $\Sigma$, respectively.

Moreover, recall that the adjoint representation of $S L(2)$, which acts on the $s l(2)$-generators $t_{\alpha}$ by conjugation, is isomorphic to the restricted Lorentz group $S O^{+}(2,1)$ in $2+1$ dimensions. This implies that the para-hypercomplex structure $\{P ; J ; \Sigma\}$ implements a global $O(2,1)$ Lorentz symmetry. See Appendix B for further details.

A $G L(2)$ rotation $(2.4)$ of the Poisson brackets $\{\cdot, \cdot\}^{a}$ induces an action "." : $G L(2) \times C^{\infty}(\mathcal{N}) \rightarrow C^{\infty}(\mathcal{N})$ on the Casimir variables

$$
\xi_{i a} \rightarrow \xi_{j b}^{\prime}=\xi_{j b}^{\prime}(g, \xi), \quad g \in G L(2),\left.\quad \xi_{j b}^{\prime}\right|_{g=\mathbf{1}_{2 \times 2}}=\xi_{j b},
$$

such that $\left\{\cdot, \xi_{a i}^{\prime}\right\}^{\prime b}$ stays diagonal in the ${ }_{a}{ }^{b}$ indices. We stress that the action (8.32) is in general not given by a linear $G L(2)$-rotation $\xi_{a i} \rightarrow \xi_{a i}^{\prime}=g_{a}{ }^{b} \xi_{b i}$, although it is indeed the case in bi-Darboux coordinates, cf. eq. (8.34). The Casimir variables $\xi_{i a}$ are in general a sort of generalized $G L(2)$ doublets in the sense of eq. (8.32), while the fiber variables $q^{i}$ are genuine $G L(2)$ singlets (=invariants).

\subsubsection{Factorizable Case}

In the factorizable case, there exists an atlas of local bi-Darboux coordinate systems (2.7), cf. Theorem 3.2. In local bi-Darboux coordinates $\left\{q^{i} ; p_{a j}\right\}$, the globally defined structures $\{$ Id; $P ; J ; \Sigma\}$ become

$\mathrm{Id}=\partial_{r}^{a j}\left(t_{0}\right)_{a}{ }^{b} \otimes \mathrm{d} \vec{p}_{b j}, \quad P=\partial_{r}^{a j}\left(t_{1}\right)_{a}{ }^{b} \otimes \mathrm{d} \vec{p}_{b j}, \quad J=\partial_{r}^{a j}\left(t_{2}\right)_{a}{ }^{b} \otimes \mathrm{d} \vec{p}_{b j}, \quad \Sigma=\partial_{r}^{a j}\left(t_{3}\right)_{a}{ }^{b} \otimes \mathrm{d} \vec{p}_{b j}$.

The formulas (8.33) are invariant under bi-canonical transformations, cf. Section 7.

A $G L(2)$ rotation (2.4) of the Poisson brackets $\{\cdot, \cdot\}^{a}$ corresponds to a $G L(2)$ rotation of the momenta

$$
p_{a i} \rightarrow p_{a i}^{\prime}=g_{a}{ }^{b} p_{b i}, \quad g \in G L(2),
$$

here written as a left group action. The $G L(2)$ rotation (8.34) induces a conjugation $t_{\alpha} \rightarrow g^{-1} t_{\alpha} g$ of the $g l(2)$-generators $t_{\alpha}$ in eq. (8.33), which in turn leads to a restricted Lorentz transformation of the para-hypercomplex structure $\{P ; J ; \Sigma\}$, cf. Appendix B.

Acknowledgement: K.B. would like to thank M. Vasiliev and the Lebedev Physics Institute for warm hospitality. The work of I.A.B. is supported by grants RFBR 11-01-00830 and RFBR 11-0200685. The work of K.B. is supported by the Grant agency of the Czech republic under the grant P201/12/G028. 


\section{A Bi-Poincaré Lemma}

\section{A.1 Algebra $\mathcal{A}$}

Consider $3 n$ coordinates $x_{\alpha}^{i}, i \in\{1, \ldots, n\}, \alpha \in\{1,2,3\}$, that are defined in an open neighborhood of the origin, and with Grassmann parity $\varepsilon\left(x_{\alpha}^{i}\right)=\varepsilon_{i}+\delta_{\alpha}^{3}$. Define three integer gradings

$$
\operatorname{deg}_{\alpha}\left(x_{\beta}^{i}\right):=\delta_{\alpha \beta}, \quad \operatorname{deg}_{\alpha}(f g)=\operatorname{deg}_{\alpha}(f)+\operatorname{deg}_{\alpha}(g), \quad f, g \in \mathbb{C}[[x]], \quad \alpha, \beta \in\{1,2,3\},
$$

and three number operators

$$
N_{\alpha}:=x_{\alpha}^{i} \frac{\overrightarrow{\partial^{\ell}}}{\partial x_{\alpha}^{i}}, \quad \alpha \in\{1,2,3\} .
$$

(No sum over $\alpha$ in the last eq. (A.2).) We will often refer to the $x_{3}^{i}$ variables as "one-forms", and the third grading " $\operatorname{deg}_{3}$ " as "form-degree". Let

$$
\mathcal{A}:=\mathbb{C}[[x]]=\bigoplus_{n_{1}, n_{2}, n_{3} \in \mathbb{N}_{0}} \mathcal{A}_{n_{1}, n_{2}, n_{3}}, \quad \mathcal{A}_{n_{1}, n_{2}, n_{3}}:=\left\{\omega \in \mathcal{A} \mid \forall \alpha \in\{1,2,3\}: \operatorname{deg}{ }_{\alpha}(\omega)=n_{\alpha}\right\},
$$

be the algebra of formal power series in the $x$ variables. A power series $\omega=\omega(x)$ of the algebra $\mathcal{A}$ will often be referred to as a "form".

\section{A.2 Differentials $d^{a}, i_{a}$ and $\mathcal{L}_{b}^{a}$}

Define 2 nilpotent and commuting Grassmann-odd differentials

$$
d^{a}:=x_{3}^{i} \frac{\overrightarrow{\partial^{\ell}}}{\partial x_{a}^{i}}, \quad \varepsilon\left(d^{a}\right)=1, \quad\left[d^{a}, d^{b}\right]=0, \quad a, b \in\{1,2\} .
$$

Define 2 dual nilpotent and commuting Grassmann-odd differentials

$$
i_{a}:=x_{a}^{i} \frac{\overrightarrow{\partial^{\ell}}}{\partial x_{3}^{i}}, \quad \varepsilon\left(i_{a}\right)=1, \quad\left[i_{a}, i_{b}\right]=0, \quad a, b \in\{1,2\} .
$$

Define their $2 \times 2=4$ mutual super-commutators

$$
\mathcal{L}_{b}^{a}:=\left[i_{b}, d^{a}\right]=x_{b}^{i} \frac{\overrightarrow{\partial^{\ell}}}{\partial x_{a}^{i}}+\delta_{b}^{a} N_{3}, \quad \varepsilon\left(\mathcal{L}_{b}^{a}\right)=0, \quad a, b \in\{1,2\} .
$$

Explicitly, they are

$$
\mathcal{L}_{1}^{1}=N_{1}+N_{3}, \quad \mathcal{L}_{2}^{2}=N_{2}+N_{3}, \quad \mathcal{L}_{1}^{2}=x_{1}^{i} \frac{\overrightarrow{\partial^{\ell}}}{\partial x_{2}^{i}}, \quad \mathcal{L}_{2}^{1}=x_{2}^{i} \frac{\overrightarrow{\partial^{\ell}}}{\partial x_{1}^{i}} .
$$

In particular, define the trace

$$
\mathcal{L}:=\mathcal{L}_{a}^{a}=\mathcal{L}_{1}^{1}+\mathcal{L}_{2}^{2}=N_{1}+N_{2}+2 N_{3} .
$$

The $\mathcal{L}_{b}^{a}$ operators form a $g l(2, \mathbb{C})$ Lie algebra,

$$
\left[\mathcal{L}_{b}^{a}, \mathcal{L}_{d}^{c}\right]=\delta_{d}^{a} \mathcal{L}_{b}^{c}-\delta_{b}^{c} \mathcal{L}_{d}^{a}, \quad a, b, c, d \in\{1,2\} .
$$

The following formulas hold

$$
\left[\mathcal{L}_{c}^{a}, d^{b}\right]=\delta_{c}^{a} d^{b}-(a \leftrightarrow b), \quad\left[i_{a}, \mathcal{L}_{b}^{c}\right]=i_{a} \delta_{b}^{c}-(a \leftrightarrow b), \quad a, b, c \in\{1,2\}
$$




\section{A.3 $\quad d$ and $i$}

Define also nilpotent second-order differential operators

$$
d:=\frac{1}{2} \epsilon_{b a} d^{a} d^{b}=d^{1} d^{2}, \quad i:=\frac{1}{2} \epsilon^{a b} i_{b} i_{a}=i_{2} i_{1}, \quad \varepsilon(d)=0, \quad \varepsilon(i)=0 .
$$

Here the sign convention for the Levi-Civita $\epsilon$-tensor is

$$
\epsilon^{a b} \epsilon_{b c}=\delta_{c}^{a}, \quad \epsilon^{12}=\epsilon_{21}=+1 .
$$

The following formulas hold

$$
\begin{gathered}
{\left[\mathcal{L}_{b}^{a}, d\right]=\delta_{b}^{a} d, \quad\left[i, \mathcal{L}_{b}^{a}\right]=\delta_{b}^{a} i, \quad a, b \in\{1,2\},} \\
{[\mathcal{L}, d]=2 d, \quad[i, \mathcal{L}]=2 i,} \\
{\left[d^{a}, i\right]=\left(\mathcal{L}_{c}^{a}+\delta_{c}^{a}\right) i_{b} \epsilon^{b c}=\mathcal{L}_{c}^{a} i_{b} \epsilon^{b c}+i_{b} \epsilon^{b a}, \quad a \in\{1,2\} .}
\end{gathered}
$$

\section{A.4 $s l(2, \mathbb{C})$ Lie Algebra}

We now decompose the four-dimensional Lie algebra $g l(2, \mathbb{C})=\mathbb{C} \oplus \operatorname{sl}(2, \mathbb{C})$. The trace operator $\mathcal{L}$ is the generator of the center $\mathbb{C}$. The three $\operatorname{sl}(2, \mathbb{C})$ generators $J_{\alpha}, \alpha \in\{1,2,3\}$, are defined as some linear combinations of the four $g l(2, \mathbb{C})$ generators $\mathcal{L}_{b}^{a}, a, b \in\{1,2\}$,

$$
\begin{array}{ll}
J_{1}:=\frac{\mathcal{L}_{1}^{2}+\mathcal{L}_{2}^{1}}{2}, & J_{2}:=\frac{\mathcal{L}_{1}^{2}-\mathcal{L}_{2}^{1}}{2 \mathrm{i}}, \quad J_{3}:=\frac{\mathcal{L}_{1}^{1}-\mathcal{L}_{2}^{2}}{2}=\frac{N_{1}-N_{2}}{2}, \\
J_{ \pm}=J_{1} \pm \mathrm{i} J_{2}, & J_{+}=\mathcal{L}_{1}^{2}, \quad J_{-}=\mathcal{L}_{2}^{1}, \quad J^{2}=J_{1}^{2}+J_{2}^{2}+J_{3}^{2} .
\end{array}
$$

It is straightforward to check that $J_{\alpha}, \alpha \in\{1,2,3\}$, form a $s l(2, \mathbb{C})$ Lie algebra,

$$
\left[J_{\alpha}, J_{\beta}\right]=\mathrm{i} \epsilon_{\alpha \beta \gamma} J_{\gamma}, \quad \epsilon_{123}=+1, \quad \alpha, \beta, \gamma \in\{1,2,3\} .
$$

Several operators commute with the $\operatorname{sl}(2, \mathbb{C})$ generators $J_{\alpha}, \alpha \in\{1,2,3\}$,

$$
\left[J^{2}, J_{\alpha}\right]=0, \quad\left[\mathcal{L}, J_{\alpha}\right]=0, \quad\left[N_{3}, J_{\alpha}\right]=0, \quad\left[d, J_{\alpha}\right]=0, \quad\left[i, J_{\alpha}\right]=0, \quad \alpha \in\{1,2,3\} .
$$

\section{A.5 Bi-Poincaré Lemma}

Lemma A.1 (Bi-Poincaré Lemma) $A d^{a}$-closed form $\omega=\omega(x), a \in\{1,2\}$, that does not contain zero- and one-forms, is locally d-exact. Or equivalently, in symbols:

$$
\forall \omega \in \mathcal{A}: \quad\left\{\begin{array}{c}
\forall a \in\{1,2\}:\left(d^{a} \omega\right)=0 \\
\operatorname{deg}_{3}(\omega) \geq 2
\end{array}\right\} \quad \Rightarrow \quad \exists \eta \in \mathcal{A}: \omega=(d \eta)
$$

Here we have defined the $\mathcal{A}$ algebra (A.3) to be the algebra $\mathbb{C}[[x]]$ of formal power series with complex coefficients. By decomposing eq. (A.20) in real and imaginary parts, it is clear that the bi-Poincaré Lemma A.1 also holds if one instead considers the algebra $\mathbb{R}[[x]]$ of formal power series with real coefficients.

Because the set $\left\{x_{1}^{1}, \ldots, x_{1}^{n} ; x_{2}^{1}, \ldots, x_{2}^{n}\right\}$ is twice as big as the set $\left\{x_{3}^{1}, \ldots, x_{3}^{n}\right\}$, we cannot apply the proof technique of e.g., Ref. [25] and Ref. [3], which requires a balanced number of variables. Instead we will use a bit of $s l(2, \mathbb{C})$ representation theory to obtain the pertinent estimate (A.37). 


\section{A.6 $L$ and $\Lambda$}

Define a third-order differential operator

$$
L:=[d, i]=\frac{1}{2} \epsilon_{b a}\left[d^{a} d^{b}, i\right]=\frac{1}{2} \epsilon_{b a}\left[d^{a},\left[d^{b}, i\right]\right]+\epsilon_{b a}\left[d^{a}, i\right] d^{b}=\Lambda+R_{b} d^{b},
$$

where

$$
R_{b}:=\epsilon_{b a} \mathcal{L}_{c}^{a} i_{d} \epsilon^{d c}, \quad b \in\{1,2\}
$$

and where

$$
\Lambda:=-\frac{1}{2} \epsilon_{b a} \mathcal{L}_{d}^{a} \mathcal{L}_{c}^{b} \epsilon^{c d}-\frac{\mathcal{L}}{2}=\frac{1}{2}\left\{\mathcal{L}_{1}^{1}, \mathcal{L}_{2}^{2}\right\}_{+}-\frac{1}{2}\left\{\mathcal{L}_{1}^{2}, \mathcal{L}_{2}^{1}\right\}_{+}-\frac{\mathcal{L}}{2}=\frac{\mathcal{L}}{2}\left(\frac{\mathcal{L}}{2}-1\right)-J^{2} .
$$

To prove the last equality in eq. (A.23), note that

$$
\frac{1}{2}\left\{\mathcal{L}_{1}^{1}, \mathcal{L}_{2}^{2}\right\}_{+}=\left(\frac{\mathcal{L}}{2}\right)^{2}-J_{3}^{2}, \quad \frac{1}{2}\left\{\mathcal{L}_{1}^{2}, \mathcal{L}_{2}^{1}\right\}_{+}=\frac{1}{2}\left\{J_{+}, J_{-}\right\}_{+}=J_{1}^{2}+J_{2}^{2} .
$$

The operators $L, \Lambda \in \operatorname{End}(\mathcal{A})$ are $g l(2, \mathbb{C})$ Casimirs,

$$
\left[\mathcal{L}_{b}^{a}, L\right]=0, \quad\left[\mathcal{L}_{b}^{a}, \Lambda\right]=0, \quad a, b \in\{1,2\}
$$

Since $\Lambda$ is a quadratic polynomial (A.23) of the four $g l(2, \mathbb{C})$ generators $\mathcal{L}_{b}^{a}$, it follows from eq. (A.25) that $L$ and $\Lambda$ commute

$$
[L, \Lambda]=0
$$

\section{A.7 Zero-Modes for $\Lambda$ ?}

Define for later convenience

$$
\Lambda^{\prime}:=\left.\Lambda\right|_{\mathcal{L} \rightarrow \mathcal{L}-2}=\left(\frac{\mathcal{L}}{2}-1\right)\left(\frac{\mathcal{L}}{2}-2\right)-J^{2}
$$

so that

$$
d f(\Lambda)=f\left(\Lambda^{\prime}\right) d, \quad f(\Lambda) i=i f\left(\Lambda^{\prime}\right),
$$

where $f=f(\lambda)$ is some function of $\lambda \in \mathbb{C}$, cf. eqs. (A.14) and (A.19).

\section{Lemma A.2}

$$
\begin{aligned}
\operatorname{ker} \Lambda \cap\left\{\omega \in \mathcal{A} \mid \operatorname{deg}_{3}(\omega) \geq 2\right\} & =\{0\} \\
\operatorname{ker} \Lambda^{\prime} \cap\left\{\omega \in \mathcal{A} \mid \operatorname{deg}_{3}(\omega) \geq 4\right\} & =\{0\} .
\end{aligned}
$$

Proof of Lemma A.2: We will only here prove the first statement (A.29), as the second statement (A.30) is similar. The vector space $\mathcal{A}$ becomes an infinite-dimensional representation of $\operatorname{sl}(2, \mathbb{C})$ by acting with the $J_{\alpha}$ generators (A.16) from the left. Since $N_{12}:=N_{1}+N_{2}=\mathcal{L}-2 N_{3}$ and $N_{3}$ are $s l(2, \mathbb{C})$ Casimirs, we only have to consider a finite-dimensional subspace

$$
\mathcal{A}_{n_{12}, n_{3}}=\left\{\omega \in \mathcal{A} \mid \operatorname{deg}_{1}(\omega)+\operatorname{deg}_{2}(\omega)=n_{12} \wedge \operatorname{deg}_{3}(\omega)=n_{3}\right\}
$$


for a pair of non-negative integers $n_{12} \in \mathbb{N}_{0} \equiv\{0,1,2, \ldots\}$ and $n_{3} \in\{2,3,4, \ldots\}$. The eigenvalue $\ell$ of the trace operator $\mathcal{L}=N_{12}+2 N_{3}$ inside $\mathcal{A}_{n_{12}, n_{3}}$ is

$$
\ell=n_{12}+2 n_{3} \text {. }
$$

The two number operators $N_{1}$ and $N_{2}$ are diagonalizable inside the pertinent subspace

$$
\mathcal{A}_{n_{12}, n_{3}}=\bigoplus_{n_{1}, n_{2} \in \mathbb{N}_{0}}^{n_{1}+n_{2}=n_{12}} \mathcal{A}_{n_{1}, n_{2}, n_{3}}
$$

with bounded eigenvalues

$$
n_{1}, n_{2} \in\left\{0,1,2, \ldots, n_{12}\right\} .
$$

According to (a superversion of) Weyl's Theorem*, a finite-dimensional representation $\mathcal{A}_{n_{12}, n_{3}}$ of a semisimple Lie algebra is always completely reducible, i.e., a finite direct sum of irreps (=irreducible representations)

$$
\mathcal{A}_{n_{12}, n_{3}}=\bigoplus_{j \in \frac{1}{2} \mathbb{N}_{0}} \mu_{j} V_{j}
$$

Here $\mu_{j} \in \mathbb{N}_{0}$ denotes the multiplicity, i.e., how many times the $(2 j+1)$-dimensional irrep $V_{j}$ appears in the direct sum (A.35), where $j \in \frac{1}{2} \mathbb{N}_{0}$ is a non-negative half-integer. Recall that the eigenvalues of $J^{2}$ and $J_{3}$ on $V_{j}$ are

$$
j(j+1) \quad \text { and } \quad m \in\{-j, 1-j, \ldots, j-1, j\},
$$

respectively. Since $\Lambda$ is a Casimir, the irrep $V_{j}$ is an eigenspace for $\Lambda$ with some eigenvalue $\lambda$, cf. Schur's Lemma. In particular, the operator $\Lambda$ is diagonalizable on the full vector space $\mathcal{A}$. We have to show that there are no zero eigenvalues $\lambda \neq 0$. Inside $V_{j} \subseteq \mathcal{A}_{n_{12}, n_{3}}$, the eigenvalues $m$ for $J_{3}=\frac{1}{2}\left(N_{1}-N_{2}\right)$ must satisfy $|m| \leq \frac{1}{2} n_{12}$, cf. eq. (A.34). In particular, this must be true for the largest eigenvalue $m=j$. Hence

$$
j \leq \frac{n_{12}}{2} .
$$

Therefore

$$
\begin{aligned}
\lambda \stackrel{(A .23)}{=} & \frac{\ell}{2}\left(\frac{\ell}{2}-1\right)-j(j+1) \\
& \geq\left(\frac{n_{12}}{2}+n_{3}\right)\left(\frac{n_{12}}{2}+n_{3}-1\right)-\frac{n_{12}}{2}\left(\frac{n_{12}}{2}+1\right)=\left(n_{12}+n_{3}\right)\left(n_{3}-1\right)>0,
\end{aligned}
$$

because $n_{12} \geq 0$ and $n_{3} \geq 2$. In particular, the operator $\Lambda$ is strictly positive.

\section{A.8 Proof of Bi-Poincaré Lemma A.1}

Proof of Bi-Poincaré Lemma A.1: $\quad$ Let there be given a $d^{a}$-closed form $\omega \in \mathcal{A}$ with $\operatorname{deg}_{3}(\omega) \geq 2$. Define a form

$$
\eta:=\left(i \Lambda^{-1} \omega\right) \stackrel{(A .28)}{=}\left(\Lambda^{\prime-1} i \omega\right),
$$

which is well-defined because of Lemma A.2. Then we calculate

$$
\begin{aligned}
& (d \eta) \stackrel{(A .39)}{=}\left(d i \Lambda^{-1} \omega\right) \stackrel{(A .21)}{=}\left(L \Lambda^{-1} \omega\right)+\left(i d \Lambda^{-1} \omega\right) \stackrel{(A .26)}{=}\left(\Lambda^{-1} L \omega\right)+\left(i d \Lambda^{-1} \omega\right) \\
& \stackrel{(A .21)+(A .28)}{=}\left(\Lambda^{-1}\left(\Lambda+R_{b} d^{b}\right) \omega\right)+\left(i \Lambda^{\prime-1} d \omega\right) \stackrel{\omega \text { closed }}{=} \omega \text {. }
\end{aligned}
$$

\footnotetext{
${ }^{*}$ It is possible to explicitly construct a sesqui-linear form $\langle\cdot, \cdot\rangle: \mathcal{A} \times \mathcal{A} \rightarrow \mathbb{C}$ that turns (the representation of) the generators $J_{\alpha}, \alpha \in\{1,2,3\}$, into Hermitian operators. This is the setting of Weyl's Theorem often stated in the Physics literature. However, Weyl's Theorem does actually not rely on the existence of any Hermitian structure, see e.g., Ref. [26].
} 


\section{B Real Lie Groups}

Here we collect some facts about the real Lie Groups $S O^{+}(2,1), S L(2)$ and $G L(2)$ used in the main text.

\section{B.1 $S O^{+}(2,1)$}

Let the Minkowski metric in $2+1$ real dimensions be

$$
\eta_{\alpha \beta}:=\left[\begin{array}{ccc}
1 & 0 & 0 \\
0 & -1 & 0 \\
0 & 0 & 1
\end{array}\right]
$$

(The non-standard ordering of spatial and temporal directions in the metric (B.1) is related to that the $\sigma_{2}$ Pauli matrix (B.13) is imaginary, cf. eq. (B.12).) The Lorentz group is

$$
O(2,1):=\left\{\Lambda \in \operatorname{Mat}_{3 \times 3}(\mathbb{R}) \mid \Lambda^{T} \eta \Lambda=\eta\right\} .
$$

The restricted Lorentz group is

$$
S O^{+}(2,1):=\left\{\Lambda \in \operatorname{Mat}_{3 \times 3}(\mathbb{R}) \mid \Lambda^{T} \eta \Lambda=\eta \wedge \operatorname{det}(\Lambda)=1 \wedge \Lambda_{2}^{2} \geq 1\right\}=e^{s o(2,1)},
$$

and its Lie algebra

$$
s o(2,1):=\left\{\lambda \in \operatorname{Mat}_{3 \times 3}(\mathbb{R}) \mid \lambda^{T}=-\eta \lambda \eta^{-1}\right\}=\operatorname{span}_{\mathbb{R}}\left\{T_{\alpha} \mid \alpha \in\{1,2,3\}\right\},
$$

with generators $T_{\alpha}, \alpha \in\{1,2,3\}$, satisfying

$$
\left[T_{\alpha}, T_{\beta}\right]=\sqrt{-\eta} \epsilon_{\alpha \beta \gamma} \eta^{\gamma \delta} T_{\delta}, \quad \alpha, \beta, \gamma, \delta \in\{1,2,3\} .
$$

Here

$$
\eta:=\operatorname{det}\left(\eta_{\alpha \beta}\right)=-1
$$

is the determinant of the Minkowski metric $\eta_{\alpha \beta}$. One may, e.g., choose generators

$$
\left(T_{\alpha}\right)_{\beta}^{\delta}=\sqrt{-\eta} \epsilon_{\alpha \beta \gamma} \eta^{\gamma \delta}, \quad \alpha, \beta, \gamma, \delta \in\{1,2,3\}
$$

so that

$$
T_{1}=\left[\begin{array}{lll}
0 & 0 & 0 \\
0 & 0 & 1 \\
0 & 1 & 0
\end{array}\right], \quad T_{2}=\left[\begin{array}{ccc}
0 & 0 & 1 \\
0 & 0 & 0 \\
-1 & 0 & 0
\end{array}\right], \quad T_{3}=\left[\begin{array}{ccc}
0 & -1 & 0 \\
-1 & 0 & 0 \\
0 & 0 & 0
\end{array}\right] \text {. }
$$

$T_{1}$ and $T_{3}$ generate Lorentz boosts, while $T_{2}$ generates spatial rotations. The Levi-Civita $\epsilon_{\alpha \beta \gamma}$-symbol satisfies

$$
\eta \epsilon_{\alpha \beta \mu} \eta^{\mu \nu} \epsilon_{\nu \gamma \delta}=\eta_{\alpha \gamma} \eta_{\beta \delta}-\eta_{\alpha \delta} \eta_{\beta \gamma}, \quad \epsilon_{123}=+1
$$




\section{B.2 $S L(2)$}

The special linear group in 2 real dimensions is

$$
S L(2):=\left\{g \in \operatorname{Mat}_{2 \times 2}(\mathbb{R}) \mid \operatorname{det}(g)=1\right\}=S p(2)=e^{s l(2)},
$$

and its Lie algebra

$$
s l(2):=\left\{x \in \operatorname{Mat}_{2 \times 2}(\mathbb{R}) \mid \operatorname{tr}(x)=0\right\}=\operatorname{span}_{\mathbb{R}}\left\{t_{\alpha} \mid \alpha \in\{1,2,3\}\right\},
$$

with generators $t_{\alpha}, \alpha \in\{1,2,3\}$, satisfying

$$
t_{\alpha} t_{\beta}=\eta_{\alpha \beta} \mathbf{1}_{2 \times 2}+\sqrt{-\eta} \epsilon_{\alpha \beta \gamma} \eta^{\gamma \delta} t_{\delta}, \quad \alpha, \beta, \gamma, \delta \in\{1,2,3\}
$$

where $\eta_{\alpha \beta}$ is the Minkowski metric (B.1) in $2+1$ dimensions. One may, e.g., choose generators

$$
t_{1}:=\sigma_{1}:=\left[\begin{array}{ll}
0 & 1 \\
1 & 0
\end{array}\right], \quad t_{2}:=-\mathrm{i} \sigma_{2}:=\left[\begin{array}{cc}
0 & -1 \\
1 & 0
\end{array}\right], \quad t_{3}:=\sigma_{3}:=\left[\begin{array}{cc}
1 & 0 \\
0 & -1
\end{array}\right] .
$$

Here $\sigma_{\alpha}, \alpha \in\{1,2,3\}$, are the Pauli matrices, which satisfies

$$
\sigma_{\alpha} \sigma_{\beta}=\delta_{\alpha \beta} \mathbf{1}_{2 \times 2}+\mathrm{i} \epsilon_{\alpha \beta \gamma} \sigma_{\gamma}, \quad \alpha, \beta, \gamma \in\{1,2,3\}
$$

\section{B.3 $G L(2)$}

The general linear group in 2 real dimensions is

$$
G L(2):=\left\{g \in \operatorname{Mat}_{2 \times 2}(\mathbb{R}) \mid \operatorname{det}(g) \neq 0\right\}=e^{g l(2)} \cong \mathbb{R}^{\times} \times S L(2), \quad \mathbb{R}^{\times}:=\mathbb{R} \backslash\{0\},
$$

where the Abelian factor $\mathbb{R}^{\times}$stores the value of the determinant $\operatorname{det}(g)$. The corresponding Lie algebra of $G L(2)$ is

$$
g l(2)=\operatorname{Mat}_{2 \times 2}(\mathbb{R})=\operatorname{End}\left(\mathbb{R}^{2}\right)=Z(g l(2)) \oplus \operatorname{sl}(2) \cong \mathbb{R} \oplus \operatorname{sl}(2) .
$$

The Lie group and Lie algebra centers are

$$
Z(G L(2))=\mathbb{R}^{\times} t_{0}, \quad Z(g l(2))=\mathbb{R} t_{0}, \quad t_{0}:=\mathbf{1}_{2 \times 2} .
$$

The $g l(2)$-generators $t_{\alpha}, \alpha \in\{0,1,2,3\}$, form the algebra (B.12) of para-quaternions, also known as the algebra of split quaternions.

\section{B.4 $S O^{+}(2,1) \cong \operatorname{Ad}(S L(2))$}

Observation B.1 The real Lie algebras so $(2,1)$ and $\operatorname{sl}(2)$ are isomorphic so $(2,1) \cong$ sl(2) via the $\operatorname{map} T_{\alpha} \mapsto \frac{1}{2} t_{\alpha}$.

Recall that the adjoint Lie group representation $\mathrm{Ad}: S L(2) \rightarrow \operatorname{End}(s l(2))$ and the adjoint Lie algebra representation ad $: \operatorname{sl}(2) \rightarrow \operatorname{End}(\operatorname{sl}(2))$ are defined as

$$
\operatorname{Ad}(g) x:=g x g^{-1}, \quad g \in S L(2), \quad x \in \operatorname{sl}(2),
$$

and

$$
\operatorname{ad}(x) y=[x, y], \quad x, y \in \operatorname{sl}(2)
$$

respectively. 
The Lie algebra $\operatorname{sl}(2)$ may be identified with Minkowski space $M(2,1) \cong \operatorname{sl}(2)$ because the determinant is the the Minkowski metric (up to a sign),

$$
\operatorname{det}(x)=\operatorname{det}\left[\begin{array}{cc}
x^{3} & x^{1}-x^{2} \\
x^{1}-x^{2} & -x^{3}
\end{array}\right]=-x^{\alpha} \eta_{\alpha \beta} x^{\beta}, \quad x=x^{\alpha} t_{\alpha} \in \operatorname{sl}(2):=\{x \mid \operatorname{tr}(x)=0\} .
$$

Since the conjugation $A d(g) x$ with an element $g \in G L(2)$ preserves traces and determinants, and hence Minkowski lengths, the group element $g$ must correspond to a Lorentz transformation $\Lambda \in O(2,1)$ of the Minkowski space $M(2,1)$. The following Proposition B.2 is a refinement of this fact.

Proposition B.2 The restricted Lorentz group $\mathrm{SO}^{+}(2,1)$ is isomorphic to the adjoint representation of $S L(2)$,

$$
S O^{+}(2,1) \cong \operatorname{Ad}(S L(2)) \cong S L(2) / \mathbb{Z}_{2} .
$$

The Lie group isomorphism is given by the map

$$
\operatorname{Ad}\left(e^{\frac{1}{2} x^{\alpha} t_{\alpha}}\right) t_{\beta}=e^{\frac{1}{2} x^{\alpha} \operatorname{ad}\left(t_{\alpha}\right)} t_{\beta}=t_{\alpha}\left(e^{x^{\gamma} T_{\gamma}}\right)_{\beta}^{\alpha}, \quad x^{\alpha} \in \mathbb{R} .
$$

In particular, $S L(2)$ is a double cover of $S O^{+}(2,1)$, because $\operatorname{Ad}\left( \pm \mathbf{1}_{2 \times 2}\right)=\mathbf{1}_{3 \times 3}$. Equation (B.22)

says in words that conjugating an $\operatorname{sl}(2)$-generator $t_{\alpha}$ with a $S L(2)$ matrix $g=e^{\frac{1}{2} x^{\alpha} t_{\alpha}}$ corresponds to a restricted Lorentz transformation $\Lambda=e^{x^{\alpha} T_{\alpha}}$ of the three $s l(2)$-generators $t_{\alpha}$. The last equality in eq. (B.22) can, e.g., be proved by scaling the variable $x^{\alpha} \rightarrow r x^{\alpha}$ with a radial 1 -parameter $r \geq 0$, and show that the left-hand side and the right-hand side satisfy the same first-order ODE with respect to the radial parameter $r$, and same initial condition at $r=0$.

\section{References}

[1] F. Magri, J. Math. Phys. 19 (1978) 1156.

[2] I.M. Gelfand and I. Zakharevich, Selecta Math. 6 (2000) 131, arXiv:math.DG/9903080.

[3] I.A. Batalin, P. Lavrov and I. Tyutin, J. Math. Phys. 31 (1990) 1487; ibid. 32 (1991) 532; ibid. 32 (1991) 2513.

[4] I.A. Batalin and R. Marnelius, Phys. Lett. B350 (1995) 44, arXiv:hep-th/9502030.

[5] I.A. Batalin, R. Marnelius and A.M. Semikhatov, Nucl. Phys. B446 (1995) 249, arXiv:hepth/9502031.

[6] P.H. Damgaard, F. De Jonghe and K. Bering, Nucl. Phys. B455 (1995) 440, arXiv:hepth/9508034.

[7] I.A. Batalin and R. Marnelius, Nucl. Phys. B465 (1996) 521, arXiv:hep-th/9510201.

[8] I.A. Batalin and G.A. Vilkovisky, Phys. Lett. 102B (1981) 27.

[9] I.A. Batalin and G.A. Vilkovisky, Phys. Rev. D28 (1983) 2567 [E: D30 (1984) 508].

[10] I.A. Batalin and G.A. Vilkovisky, Nucl. Phys. B234 (1984) 106.

[11] I.A. Batalin and G.A. Vilkovisky, J. Math. Phys. 26 (1985) 172. 
[12] M.A. Grigoriev and A.M. Semikhatov, Phys. Lett. B417 (1998) 259, arXiv:hep-th/9708077.

[13] M.A. Grigoriev and A.M. Semikhatov, Theor. Math. Phys. 124 (2000) 1157, arXiv:hepth/9807023.

[14] M. Obata, Japan J. Math. 26 (1956) 43.

[15] A.S. Dancer, H.R. Jørgensen and A.F. Swann, Rend. Semin. Mat. Torino 63 (2005) 119, arXiv:math.DG/0412215.

[16] A. Andrada, Annals of Global Analysis and Geometry 27 (2005) 377.

[17] D.V. Alekseevsky and V. Cortés, Osaka J. Math. 45 (2008) 215.

[18] D.V. Alekseevsky, C. Medori and A. Tomassini, Russian Math. Surveys 64 (2009) 1, arXiv:0806.2272.

[19] M. Göteman and U. Lindström, Lett. Math. Phys. 95 (2011) 211, arXiv:0903.2376.

[20] M.A. Grigoriev, Phys. Lett. B458 (1999) 499, arXiv:hep-th/9901046.

[21] P. Libermann and C. Marle, Symplectic Geometry and Analytical Mechanics, Springer, 1987.

[22] H. Goldstein, Classical Mechanics, 2nd ed., Reading, Massachusetts, Addison-Wesley Publishing, 1980 .

[23] J. Moser, On the volume elements on a manifold, Trans. Amer. Math. Soc. 120 (1965) 286-294.

[24] K. Bering, Almost parity structure, connections and vielbeins in BV geometry, arXiv:physics/9711010.

[25] I.A. Batalin, P.M. Lavrov and I.V. Tyutin, J. Math. Phys. 31 (1990) 6; ibid. 31 (1990) 2708; Int. J. Mod. Phys. A 6 (1991) 3599.

[26] J.E. Humphreys, Introduction to Lie Algebras and Representation Theory, Springer, 1972. 